MATHEMATICS OF COMPUTATION

Volume 66, Number 219, July 1997, Pages 1239-1267

S 0025-5718(97)00852-1

\title{
COMPUTING STARK UNITS FOR TOTALLY REAL CUBIC FIELDS
}

\author{
DAVID S. DUMMIT, JONATHAN W. SANDS, AND BRETT A. TANGEDAL
}

\begin{abstract}
A method for computing provably accurate values of partial zeta functions is used to numerically confirm the rank one abelian Stark Conjecture for some totally real cubic fields of discriminant less than 50000. The results of these computations are used to provide explicit Hilbert class fields and some ray class fields for the cubic extensions.
\end{abstract}

\section{INTRODUCTION}

Let $K / k$ be an abelian extension of number fields with Galois group $G=$ $\operatorname{Gal}(K / k)$ and let $S$ be a finite set of places of $k$ that includes the Archimedean places of $k$ and all the places of $k$ ramified in $K / k$. For any $\sigma \in G$ the partial zeta function $\zeta_{S}(s, \sigma)$ is defined for $\operatorname{Re}(s)>1$ by

$$
\zeta_{S}(s, \sigma)=\sum_{\substack{\mathfrak{A} \text { integral } \\(\mathfrak{A}, S)=1 \\ \sigma \mathfrak{A}=\sigma}} \frac{1}{\mathbf{N A}^{s}}
$$

where the sum extends over all integral ideals $\mathfrak{A}$ of $k$ whose Frobenius symbol $\sigma_{\mathfrak{A}}$ for the abelian extension $K / k$ is the given element $\sigma$ of $G$.

For a character $\chi$ of $G$ define $L_{S}(s, \chi)$ to be the $L$-series for $\chi$ with the Euler factors at $S$ removed, which for $\operatorname{Re}(s)>1$ is given by the usual convergent Euler product:

$$
L_{S}(s, \chi)=\prod_{(\mathfrak{p}, S)=1}\left(1-\frac{\chi(\mathfrak{p})}{\mathbf{N p}^{s}}\right)^{-1}
$$

where $\chi(\mathfrak{p})=\chi\left(\sigma_{\mathfrak{p}}\right)$ and the product is taken over all primes $\mathfrak{p}$ of $k$ not in the set $S$.

Both $\zeta_{S}(s, \sigma)$ and $L_{S}(s, \chi)$ have meromorphic continuations to the entire $s$-plane. The order $r(\chi)$ of the zero of $L_{S}(s, \chi)$ at $s=0$ is one less than the number of places in $S,|S|-1$, if $\chi=1$ is the trivial character; and $r(\chi)$ is the number of places $v$ in $S$ for which $\chi$ is trivial on the decomposition group of $v$ for the extension $K / k$ if $\chi$ is not trivial.

In particular, suppose $S$ contains at least two places, including one place $v$ that splits completely in $K$. Then every $L_{S}(s, \chi)$ has a zero of order at least one at $s=0$ and it follows that the same is true of the partial zeta functions. The abelian Stark

Received by the editor February 9, 1996 and, in revised form, May 15, 1996.

1991 Mathematics Subject Classification. Primary 11R42; Secondary 11Y40, 11R37, 11R16 .

Research supported by grants from the NSA and the NSF.

(C)1997 American Mathematical Society 
Conjecture, the first inklings of which appear in [20] and which was developed in the fundamental series of papers [22]-[25], is that the coefficient of $s$ in the Taylor expansions of these zeta functions near $s=0$ can be computed 'algebraically':

Rank One Abelian Stark Conjecture. Under the hypotheses on $K / k$ and $S$ above, there exists an $S$-unit $\epsilon$ in $K$ such that

- If $S$ contains at least 3 places, then $|\epsilon|_{w^{\prime}}=1$ for all places $w^{\prime}$ of $K$ not dividing the place $v$ of $k$. In particular, $\epsilon$ is a $v$-unit in $K$.

If $S=\left\{v, v^{\prime}\right\}$, then $|\epsilon|_{\sigma w^{\prime}}=|\epsilon|_{w^{\prime}}$ for all $\sigma \in G$ and all places $w^{\prime}$ of $K$ dividing $v^{\prime}$.

- $\log \left|\epsilon^{\sigma}\right|_{w}=-e \zeta_{S}^{\prime}(0, \sigma)$ for all $\sigma \in G$, where $e$ is the number of roots of unity in $K$, where $w$ is a prime of $K$ lying above the prime $v$ of $k$ that splits completely in $K$, and where the absolute values are normalized. Equivalently, $L_{S}^{\prime}(0, \chi)=-\frac{1}{e} \sum_{\sigma \in G} \chi(\sigma) \log \left|\epsilon^{\sigma}\right|_{w}$ for all characters $\chi$ of $G$.

- The extension $K\left(\epsilon^{1 / e}\right)$ is an abelian extension of $k$.

Remark. The statement above is stronger than the more conservative versions actually conjectured (in print) by Stark, specifically with respect to the last abelian condition. The version above appears in [26]. A generalization of this Conjecture (again for abelian extensions $K / k$ ) to higher-order zeros appears in [14], which is the reason we refer to this as the rank one situation.

The purpose of this paper is to consider this conjecture in one of the first situations for which it is not known, namely the situation of a totally real cubic base field $k$ (the first section below indicates those situations in which the conjecture has been proved). This work was motivated by a computation of Stark. The methods used to compute the relevant values of the partial zeta functions, described in Section 3, considerably improve upon Stark's original computations. In Section 4 we use the results of these computations to indicate how Stark's conjecture can be used to compute explicit Hilbert class fields for some totally real cubic fields, very much in the spirit of Stark's interpretation of his conjecture as a step in the direction of solving Hilbert's 12th Problem. The complete numerical confirmation of Stark's conjecture for these cubic fields (to the accuracy of the computations, generally $10^{-30}$ ) is done in Section 5 . An explicit interesting example is described in detail in Section 6 and the final section contains some relevant tables.

\section{Preliminaries}

For a given abelian extension $K / k$, the hypotheses on the set $S$ for the abelian Stark Conjecture $\operatorname{St}(K / k, S)$ of the previous section are that (1) $S$ contains all the Archimedean primes of $k$ and all the finite primes of $k$ ramifying in the extension $K / k$, (2) $S$ contains at least two primes, and (3) $S$ contains at least one prime $v$ decomposing completely in $K / k$. In this paper we shall be concerned with the situation where $k$ is a totally real cubic field, $K$ is a certain abelian extension of $k$ unramified at all finite primes of $k$, and $S$ consists precisely of the Archimedean primes of $k$. We first collect the known results regarding $\operatorname{St}(K / k, S)$ in this section (note, however, that the function field and local conjectures are not considered, nor are the connections with Kolyvagin Euler systems), and indicate the status of this Conjecture. The totally real cubic fields considered later are one of the first cases where the rank one Stark conjecture $\operatorname{St}(K / k, S)$ has not been proved. 
(i) If $S$ contains at least two primes totally split in $K$, then $\operatorname{St}(K / k, S)$ is true (for more or less trivial reasons: For $\chi \neq 1, L(s, \chi)$ has a zero at $s=0$ of order at least 2 , so we can take $\epsilon=1$ if $S$ has at least 3 elements. It remains to consider the case $\chi=1$ and $S$ of cardinality 2, cf. [26], p. 91).

(ii) In particular, if $S$ contains at least 2 complex Archimedean primes, then $\operatorname{St}(K / k, S)$ is true, [26], p. 92 .

(iii) If $\operatorname{St}(K / k, S)$ is true, then $\operatorname{St}\left(K / k, S^{\prime}\right)$ is true for any $S \subseteq S^{\prime}$ (by induction: $S^{\prime}=S \cup\left\{v^{\prime}\right\}$ so $\epsilon_{S^{\prime}}=\epsilon^{1-F_{v}}$ where $F_{v}$ is the Frobenius for $v$, cf. [26], p. 92).

(iv) If $\operatorname{St}(K / k, S)$ is true, then $\operatorname{St}\left(K^{\prime} / k, S\right)$ is true for $k \subseteq K^{\prime} \subseteq K$, with unit given up to a root of unity by the norm: $\zeta N_{K / K^{\prime}}(\epsilon)$ (this follows essentially formally from the functorial properties of the $L$-series, cf. [26], p. 92). See also [12].

(v) If $k$ is totally real and $v$ is a fixed (real) Archimedean place of $k$, then the field obtained by adjoining all Stark units $\epsilon$ as $K$ varies over fields in which $v$ splits completely is essentially the full abelian closure of the embedding of $k$ in $\mathbb{R}$ defined by $v$ ([26], p. 94).

(vi) $\operatorname{St}(K / k, S)$ is true if $k=\mathbb{Q}$ or if $k$ is an imaginary quadratic field ([25], [26], p. 95 , cf. also [7]-[9]).

(vii) $\operatorname{St}(K / k, S)$ is true if $|S|=2$, ([26], p. 98).

(viii) $\operatorname{St}(K / k, S)$ is true if $[K: k]=2$ (this follows from an analysis of the ' \pm parts' of the classical formula for the residue at $s=0$ of the Dedekind zeta functions ([26], p. 104).

(ix) $\mathrm{St}(K / k, S)$ is true if $\operatorname{Gal}(K / k)$ is of exponent 2 and order $2^{m}$ with either $K / k$ tamely ramified or $|S|>m+1$, ([16], [17]).

(x) $\operatorname{St}(K / k, S)$ is true if $v$ is a finite prime, $K$ is abelian over $\mathbb{Q}$ and $S$ contains all primes dividing the discriminant of $K$ over $\mathbb{Q}([15])$.

(xi) If $k$ is a totally real field and $K$ is a CM field, then, under some mild additional assumptions on the set $S$ depending on the field $k$, the first two parts of $\operatorname{St}(K / k, S \cup\{v\})$ above (the "annihilation portion of the Brumer Conjecture") are true for all finite primes $v$ whose order in the class group of $k$ is odd ([28]).

By (iii) it suffices, for a given abelian extension $K / k$, to verify $\operatorname{St}(K / k, S)$ with a minimal set $S$, i.e., for $S$ consisting precisely of the Archimedean primes of $k$ and the primes of $k$ ramified in $K$. The minimal possible such set $S$ would consist precisely of the Archimedean primes of $k$, which leads to a consideration of abelian extensions $K / k$ that are unramified at all the finite primes of $k$. By (ii) we may take $k$ either totally real or having precisely one complex Archimedean prime, and we may also assume that precisely one Archimedean prime of $k$ splits completely in $K$. If this Archimedean prime is real, then all remaining real infinite primes of $k$ become complex in $K$ and this is the situation considered in this paper. (Note that to date no computations have been done in the situation where there is more than one Archimedean prime of $k$ and the unique Archimedean prime of $k$ splitting in $K$ is complex.) Then, by (iv), we may assume that $K$ is the maximal abelian extension $K$ of $k$ subject to these constraints.

By (vi) the minimal fields $k$ for which $\operatorname{St}(K / k, S)$ is not known to be true are real quadratic fields $k$ and fields $k$ with $[k: \mathbb{Q}]=3$. Stark considered the situation of real quadratic fields, publishing several examples but computing a number of others (also C. Fogel, [5], has computed a number of (unpublished) examples in 
this case). Shintani, in [18] and [19], found some special cases of the Stark conjecture and computed the values of derivatives of certain $L$-series for real quadratic fields in terms of double $\Gamma$-functions, in particular computing a number of explicit examples (cf. also [1], [13], and [17] for some special cases). Hayes, in [10] and [11], has considered the real quadratic case in the situation where the prime $v$ of $k$ splitting in $K$ is non-Archimedean. The next case to consider concerns cubic extensions $k$ of $\mathbb{Q}$ which are either totally real (with one real Archimedean prime $\mathfrak{p}_{\infty}^{(1)}$ splitting completely in $K$ and the other two ramifying) or have precisely one complex Archimedean prime (with the real prime ramifying in $K$ ).

In this paper we consider the case where $k$ is a totally real cubic field with Archimedean primes $\mathfrak{p}_{\infty}^{(1)}, \mathfrak{p}_{\infty}^{(2)}$, and $\mathfrak{p}_{\infty}^{(3)}$.

By the remarks above, the field $K$ should be taken to be the maximal abelian extension of $k$ unramified outside $\mathfrak{p}_{\infty}^{(2)}$ and $\mathfrak{p}_{\infty}^{(3)}$ (i.e., $K$ is the ray class field of $k$ of conductor $\left.\mathfrak{p}_{\infty}^{(2)} \mathfrak{p}_{\infty}^{(3)}\right)$ and both $\mathfrak{p}_{\infty}^{(2)}$ and $\mathfrak{p}_{\infty}^{(3)}$ should ramify in $K$. The latter condition implies in particular that $K$, which contains the Hilbert class field $H$ of $k$, should be in fact a proper extension of $H$.

Because of the results of (viii) and (ix), it is of interest to consider the case where the class number of $k$ is divisible by 3. Stark, in [21] and [25], (cf. also [26], pp. 98-102) considered one numerical example of such a totally real cubic field having class number 3. In Stark's example the Hilbert class field $H$ is obtained as the composite with $k$ of an abelian extension of $\mathbb{Q}$ of degree 3 (i.e., $H$ is a genus field over $k$ ). Discussion of this example between Stark and the first author a few years ago suggested consideration of a field $k$ for which $H$ is not a genus field, which led to the investigations of this paper. The first totally real cubic field with class number divisible by 3 for which the associated Hilbert class field is not a genus field has discriminant $D_{k}=28212$. This field is considered in some detail in Section 6 below.

\section{Computing Derivatives of the PARTial ZEta FunCtions}

For the reasons indicated in the previous section, let $k$ be a totally real cubic field of class number 3 and let $S$ consist of the Archimedean primes $\left\{\mathfrak{p}_{\infty}^{(1)}, \mathfrak{p}_{\infty}^{(2)}, \mathfrak{p}_{\infty}^{(3)}\right\}$ of $k$. Denote by $D_{k}$ (resp., $\mathfrak{D}_{k}$ ) the discriminant (resp., different) of $k$ over $\mathbb{Q}$. Let $H$ be the Hilbert class field to $k$ and let $K$ be the ray class field to $k$ corresponding to the conductor $\mathfrak{p}_{\infty}^{(2)} \mathfrak{p}_{\infty}^{(3)}$, with Galois group $G=\operatorname{Gal}(K / k)$. The ray class group of conductor $\mathfrak{p}_{\infty}^{(2)} \mathfrak{p}_{\infty}^{(3)}$ is isomorphic to $G$ under the Artin map which maps the class $\mathfrak{b}$ to the Frobenius symbol $\sigma_{\mathfrak{b}}=\sigma_{\mathfrak{A}}$ for any fractional ideal $\mathfrak{A}$ in the class $\mathfrak{b}$.

We assume that $K$ is a proper extension of $H$, i.e., that $[K: H]=2$ (as indicated, this avoids the trivial situation (i) of Section 2), and let $\tau$ be a generator for the Galois group $\operatorname{Gal}(K / H)$ (so that $\tau$ generates the decomposition group in $K / k$ for both $\mathfrak{p}_{\infty}^{(2)}$ and $\left.\mathfrak{p}_{\infty}^{(3)}\right)$. For any ray class $\mathfrak{b}$ of $k$ let $\overline{\mathfrak{b}}$ denote the class with $\sigma_{\overline{\mathfrak{b}}}=\tau \sigma_{\mathfrak{b}}$ in $G$.

For simplicity let $\zeta(s, \mathfrak{b})=\zeta_{S}\left(s, \sigma_{\mathfrak{b}}\right)$. Define (following Stark [21],[24]):

$$
\Lambda_{\mathfrak{b}}(s)=\left(\sqrt{\frac{D_{k}}{\pi^{3}}}\right)^{s} \Gamma\left(\frac{s}{2}\right) \Gamma\left(\frac{s+1}{2}\right)^{2}[\zeta(s, \mathfrak{b})-\zeta(s, \overline{\mathfrak{b}})] .
$$


The function $\Lambda_{\mathfrak{b}}(s)$ is entire and taking the limit as $s$ tends to 0 shows that

$$
\Lambda_{\mathfrak{b}}(0)=2 \pi\left[\zeta^{\prime}(0, \mathfrak{b})-\zeta^{\prime}(0, \overline{\mathfrak{b}})\right] .
$$

Since $\tau$ generates the decomposition group for both Archimedean primes $\mathfrak{p}_{\infty}^{(2)}$ and $\mathfrak{p}_{\infty}^{(3)}$, for every character $\chi$ of $G$ with $\chi(\tau)=1$ the corresponding $L$-series $L(s, \chi)=$ $L_{S}(s, \chi)$ has a zero at $s=0$ of order at least 2 . It follows that

$$
\zeta(s, \mathfrak{b})+\zeta(s, \overline{\mathfrak{b}})=\frac{1}{3} \sum_{\substack{\chi(\tau)=1 \\ \chi \in \hat{G}}} \bar{\chi}(\mathfrak{b}) L(s, \chi)
$$

also has a zero of order at least 2 at $s=0$. Hence $\zeta^{\prime}(0, \mathfrak{b})+\zeta^{\prime}(0, \overline{\mathfrak{b}})=0$ and it follows that

$$
\Lambda_{\mathfrak{b}}(0)=4 \pi \zeta^{\prime}(0, \mathfrak{b})
$$

This formula allows us to reduce the computation of the values at zero of the derivatives of the partial zeta functions involved in Stark's Conjecture to the computation of the value at zero of $\Lambda_{\mathfrak{b}}(s)$. In Stark's original computations, these values were computed as values of a triple integral of a three-dimensional theta function. The numerical evaluation of these integrals allowed only a limited accuracy and involved in particular the use of an iterative integration scheme which is not proved to converge to the correct value.

As indicated in the Introduction, one of the purposes of the computation of the Stark unit is to use this numerical information to recognize the conjectured algebraic integers and then to use these algebraic integers to construct the relevant ray class fields. For this purpose, much higher accuracy than Stark's original computations is required. Rather than compute the values $\Lambda_{\mathfrak{b}}(0)$ as iterated integrals, we instead compute them in terms of certain line integrals, which allows for increased accuracy and has the additional advantage of providing provably accurate results. This method was first introduced to us by E. Friedman and the argument leading to Proposition 1 below is from his paper [6].

The analytic function $\Lambda_{\mathfrak{b}}(s)$ satisfies the functional equation

$$
\Lambda_{\mathfrak{b}}(1-s)=-\Lambda_{\mathfrak{b}}(s)
$$

where the 'dual' ray class $\check{\mathfrak{b}}$ is the class $\mathfrak{b}^{-1}\left[\mathfrak{D}_{k}\right]$ in the ray class group of conductor $\mathfrak{p}_{\infty}^{(2)} \mathfrak{p}_{\infty}^{(3)}$

For simplicity let $\Lambda(s)=\Lambda_{\mathfrak{b}}(s)$ and $\check{\Lambda}(s)=\Lambda_{\mathfrak{b}}(s)$. Define

$$
\Lambda_{+}(z)=\Lambda(z)-\check{\Lambda}(z), \quad \Lambda_{-}(z)=\Lambda(z)+\check{\Lambda}(z)
$$

and

$$
\rho_{ \pm}(s, z)=\frac{1}{z-s} \pm \frac{1}{(1-z)-s} .
$$

The line integral

$$
I_{ \pm}(s)=\frac{1}{2 \pi i} \int_{\delta-i \infty}^{\delta+i \infty} \Lambda_{ \pm}(z) \rho_{\mp}(s, z) d z
$$

defines an analytic function of $s$ that is independent of the choice of $\delta$ in the region $\max (\operatorname{Re}(s), \operatorname{Re}(1-s))<\delta$. If $1 / 2<\operatorname{Re}(s)<\delta$, then the analyticity of the function 
$\Lambda(z)$ implies that moving the line of integration left to $\delta=1 / 2$ picks up only the residue at $z=s$ and shows

$$
I_{ \pm}(s)=\Lambda_{ \pm}(s)+\frac{1}{2 \pi i} \int_{1 / 2-i \infty}^{1 / 2+i \infty} \Lambda_{ \pm}(z) \rho_{\mp}(s, z) d z .
$$

The functional equation for $\Lambda(s)$ shows that $\Lambda_{ \pm}\left(\frac{1}{2}+i t\right)=\Lambda\left(\frac{1}{2}+i t\right) \pm \Lambda\left(\frac{1}{2}-i t\right)$. It follows that $\Lambda_{ \pm}(z) \rho_{\mp}(s, z)$ is antisymmetric along the line $\operatorname{Re}(z)=1 / 2$, so that the integral along this line vanishes by symmetry. This shows

$$
I_{ \pm}(s)=\Lambda_{ \pm}(s)
$$

for all $s$ with $1 / 2<\operatorname{Re}(s)<\delta$. It follows that the same equality holds for all $s$ with $\max (\operatorname{Re}(s), \operatorname{Re}(1-s))<\delta$ by analytic continuation provided $\delta>\frac{1}{2}$. Hence

$$
\Lambda_{\mathfrak{b}}(s)=\frac{1}{2}\left(\Lambda_{+}(s)+\Lambda_{-}(s)\right)=\frac{1}{2}\left(I_{+}(s)+I_{-}(s)\right)
$$

provided the line integrals are computed with a $\delta$ with $\delta>\max \left(\frac{1}{2}, \operatorname{Re}(s), \operatorname{Re}(1-s)\right)$.

For a fixed ray class $\mathfrak{b}$ of $k$, let $a_{n}(\mathfrak{b})$ denote the number of integral ideals of $k$ of absolute norm equal to $n$ lying in the class $\mathfrak{b}$ and define similarly $a_{n}(\overline{\mathfrak{b}}), a_{n}(\check{\mathfrak{b}})$ and $a_{n}(\overline{\mathfrak{b}})$.

Define

$$
\begin{aligned}
& A_{n}=a_{n}(\mathfrak{b})-a_{n}(\overline{\mathfrak{b}}), \\
& B_{n}=a_{n}(\check{\mathfrak{b}})-a_{n}(\overline{\mathfrak{b}})
\end{aligned}
$$

so that $\zeta(s, \mathfrak{b})-\zeta(s, \overline{\mathfrak{b}})=\sum_{n=1}^{\infty} A_{n} / n^{s}$ and the $B_{n}$ are the coefficients for the Dirichlet series for the corresponding dual class.

Proposition 1. With notations as above,

$$
\begin{aligned}
4 \pi \zeta^{\prime}(0, \mathfrak{b})=\Lambda_{\mathfrak{b}}(0)=\sum_{n=1}^{\infty} & {\left[A_{n} \frac{1}{2 \pi i} \int_{\frac{1}{2}-i \infty}^{\frac{1}{2}+i \infty}\left(\frac{\sqrt{D_{k} / \pi^{3}}}{n}\right)^{z} \Gamma\left(\frac{z}{2}\right) \Gamma\left(\frac{z+1}{2}\right)^{2} \frac{d z}{z}\right.} \\
& \left.-B_{n} \frac{1}{2 \pi i} \int_{\frac{3}{2}-i \infty}^{\frac{3}{2}+i \infty}\left(\frac{\sqrt{D_{k} / \pi^{3}}}{n}\right)^{z} \Gamma\left(\frac{z}{2}\right) \Gamma\left(\frac{z+1}{2}\right)^{2} \frac{d z}{z-1}\right] .
\end{aligned}
$$

Proof. Applying equation (2) and the definition of $I_{ \pm}(s)$ with $\delta=3 / 2$ gives

$$
\begin{aligned}
\Lambda_{\mathfrak{b}}(0) & =\frac{1}{2 \pi i} \int_{\frac{3}{2}-i \infty}^{\frac{3}{2}+i \infty}\left(\frac{\Lambda(z)}{z}+\frac{\check{\Lambda}(z)}{1-z}\right) d z \\
& =\frac{1}{2 \pi i} \int_{\frac{1}{2}-i \infty}^{\frac{1}{2}+i \infty} \frac{\Lambda(z)}{z} d z-\frac{1}{2 \pi i} \int_{\frac{3}{2}-i \infty}^{\frac{3}{2}+i \infty} \frac{\check{\Lambda}(z)}{z-1} d z .
\end{aligned}
$$

Using the series expansions for $\Lambda(z)$ and $\check{\Lambda}(z)$ and interchanging summation and integration gives the formula in the proposition.

By the proposition, the accurate computation of the values $\zeta^{\prime}(0, \mathfrak{b})$ to high precision has been reduced to the accurate computation of line integrals essentially 
independent of the field $k$ of the form

$$
F(a)=\frac{1}{2 \pi i} \int_{\frac{1}{2}-i \infty}^{\frac{1}{2}+i \infty} a^{z} \Gamma\left(\frac{z}{2}\right) \Gamma\left(\frac{z+1}{2}\right)^{2} \frac{d z}{z} .
$$

These are computed by shifting the line of integration to the left and computing the residues:

$$
\begin{gathered}
F(a)=\pi(-3 \gamma-4 \ln 2+2 \ln a)+\sum_{j=1}^{J}\left(\rho_{j, 1}+\rho_{j, 2} \ln a\right) a^{-j}+ \\
\frac{1}{2 \pi i} \int_{-J-\frac{1}{2}-i \infty}^{-J-\frac{1}{2}+i \infty} a^{z} \Gamma\left(\frac{z}{2}\right) \Gamma\left(\frac{z+1}{2}\right)^{2} \frac{d z}{z}
\end{gathered}
$$

where $J \geq 1$ is an integer. Here $\gamma$ is Euler's gamma constant and

$$
\begin{aligned}
& \rho_{j, 2}= \begin{cases}\frac{\sqrt{\pi}(-1)^{(j-1) / 2} 2^{j+2}}{j\left(\frac{j-1}{2}\right) ! j !} & (j \text { odd }), \\
0 & (j \text { even }),\end{cases} \\
& \rho_{j, 1}= \begin{cases}\rho_{j, 2}\left(\frac{1}{j}+\frac{-3 \gamma+H_{(j-1) / 2}+2 H_{j}-2 \ln 2}{2}\right) & (j \text { odd }), \\
\frac{\pi(-1)^{1+j / 2} 4^{j}(j / 2-1) !}{(j !)^{2}} & (j \text { even })\end{cases}
\end{aligned}
$$

(with $H_{m}=1+1 / 2+\ldots+1 / m$ ) are residues arising from the gamma function factors.

Number of integrals to compute. The estimate

$$
|F(a)| \sim \frac{2 \pi}{\sqrt{3}} a^{2 / 3} e^{-3 a^{-2 / 3}}
$$

for the integrals used in computing $\zeta^{\prime}(0, \mathfrak{b})$ (cf. [6], Prop. 2.3 and [2]) leads to the following estimate for the error obtained summing $N$ terms:

$$
20 D_{k}^{2 / 3} e^{-\left(3 \pi / D_{k}{ }^{1 / 3}\right) N^{2 / 3}} \text {. }
$$

It follows that to provide an accuracy of at least $10^{-M}$, it suffices to take

$$
N>0.18 \sqrt{D_{k}}\left(1+0.77 M+\frac{2}{9} \ln D_{k}\right)^{3 / 2}
$$

for the number of integral terms to compute.

Number of residues to compute. The integral along the line $\operatorname{Re}(z)=-J-1 / 2$ is easily estimated by Stirling's formula to be at most

$$
\left|\frac{1}{2 \pi i} \int_{-J-\frac{1}{2}-i \infty}^{-J-\frac{1}{2}+i \infty} a^{z} \Gamma\left(\frac{z}{2}\right) \Gamma\left(\frac{z+1}{2}\right)^{2} \frac{d z}{z}\right|<\frac{8}{\sqrt{\pi^{3} a J}}\left(\frac{8 e^{3} a^{-2}}{J^{3}}\right)^{J / 2}
$$

For a given accuracy $10^{-M}$ it is an easy matter to compute the number of residues to compute for a given value of $a$. The following table indicates some typical values of $J$ corresponding to a given $a$ to insure an accuracy of at least $10^{-M}$ : 


$\begin{array}{lrrr}a \backslash M & 16 & 32 & 48 \\ & & & \\ 100 & 7 & 13 & 17 \\ 10 & 11 & 18 & 24 \\ 1 & 20 & 29 & 38 \\ 10^{-1} & 44 & 59 & 71 \\ 10^{-2} & 140 & 160 & 178 \\ 10^{-3} & 569 & 592 & 614 \\ 10^{-4} & 2549 & 2573 & 2597\end{array}$

It is straightforward to show that the individual residue contribution $\left(\rho_{j, 1}+\rho_{j, 2} \ln a\right) a^{-j}$ to the sum for the integral $F(a)$ is maximal for $j \sim 2 a^{-2 / 3}$ with value approximately $a e^{3 a^{-2 / 3}}$ when $j$ is even and for $j \sim 2 a^{-2 / 3} \pm a^{-1 / 3}$ with value approximately $a^{4 / 3} e^{3 a^{-2 / 3}}$ when $j$ is odd. Note that these maximal values are approximately the reciprocals of the values of the integrals $F(a)$ being computed, so that typically it is necessary to sum a number of very large residues to accurately compute the very small line integrals of interest. In particular, this means that in computations with a final desired accuracy of at least $10^{-M}$ the internal computations must be done with an accuracy of at least $10^{-2 M}$.

Example. For Stark's explicit cubic example $\mathbb{Q}(\alpha), \alpha^{3}-\alpha^{2}-9 \alpha+8=0$, with $D_{k}=2597$ (cf. [21], pp. 1073-4, and [26], pp. 98-102), the choices $N=1250$, $J=200$, and an internal computation precision of $10^{-70}$, computes the values of the zeta functions to a proved accuracy of at least $10^{-30}$ :

$$
\begin{aligned}
& 2 \zeta^{\prime}\left(0, \mathfrak{c}^{0}\right)=-2 \zeta^{\prime}\left(0, \mathfrak{c}^{3}\right)=2.6229258798145544647221697471032 \ldots \\
& 2 \zeta^{\prime}\left(0, \mathfrak{c}^{1}\right)=-2 \zeta^{\prime}\left(0, \mathfrak{c}^{4}\right)=0.5567427719936224099615684806856 \ldots \\
& 2 \zeta^{\prime}\left(0, \mathfrak{c}^{2}\right)=-2 \zeta^{\prime}\left(0, \mathfrak{c}^{5}\right)=-0.7266809196046131328605974037158 \ldots
\end{aligned}
$$

(here $\mathfrak{c}$ is the ray class containing the prime ideal $\mathfrak{p}=(2, \beta)$ ). The values agree with the values predicted by Stark's units to $10^{-30}$, and agree with the values computed by Stark to $10^{-16}, 10^{-15}$ and $10^{-15}$, respectively. Note that the values computed by Stark were not proved to be correct (but now have been, by these computations).

The 'residue packets' for the line integrals above are themselves very interesting. As an example, in recomputing the values for Stark's example above, one encounters the following line integral (corresponding to a term with $n=994$ ):

$$
\frac{1}{2 \pi i} \int_{1 / 2-i \infty}^{1 / 2+i \infty}\left(\frac{\sqrt{2597 / \pi^{3}}}{994}\right)^{z} \Gamma\left(\frac{z}{2}\right) \Gamma\left(\frac{1+z}{2}\right)^{2} \frac{d z}{z},
$$

which has value approximately $6.95702479\left(10^{-31}\right)$. The plot in Figure 1 indicates the residues used in computing this value and indicates the reciprocal correlation between the value of the line integral being computed and the maximum residue involved (and also indicates the 'packet' nature of the data). 


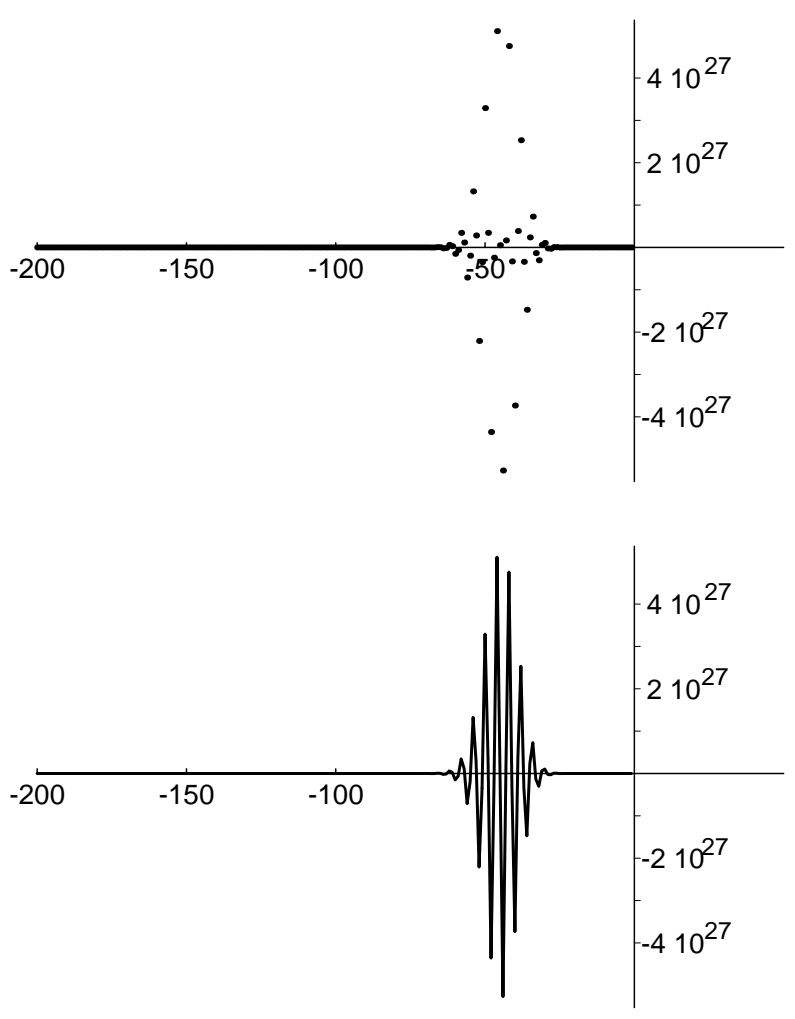

FiguRE 1

\section{Numerical Confirmation of Stark's Conjecture}

We continue with the hypotheses on $k, S$ and $K$ in Section $3: k$ is a totally real cubic field with class number $3, S=\left\{\mathfrak{p}_{\infty}^{(1)}, \mathfrak{p}_{\infty}^{(2)}, \mathfrak{p}_{\infty}^{(3)}\right\}$ consists of the Archimedean primes of $k, K$ is the ray class field to conductor $\mathfrak{p}_{\infty}^{(2)} \mathfrak{p}_{\infty}^{(3)}$, and $[K: k]=6$.

There are 113 totally real cubic fields $k$ with class number divisible by 3 and having discriminant $D_{k}$ less than 50000 (from the tables [4]). Each cubic field $k$ is given explicitly as $\mathbb{Q}(\beta)$ where $\beta$ is a root of an irreducible cubic polynomial $f(x)$. The specification of the place $\mathfrak{p}_{\infty}^{(1)}$ amounts to a choice $\beta_{1}$ of one of the three real roots of $f(x)$ to define an embedding of $k$ into $\mathbb{R}$. Computing the orders of the appropriate ray class groups shows that, for a given choice of embedding defining $\mathfrak{p}_{\infty}^{(1)}$, the signs of the fundamental units of $k$ in the other two embeddings determine whether the ray class field $K$ to conductor $\mathfrak{p}_{\infty}^{(2)} \mathfrak{p}_{\infty}^{(3)}$ is strictly larger than the Hilbert class field of $k$ : the condition translates into the condition that the product of the signs of the two fundamental units at $\mathfrak{p}_{\infty}^{(2)}$ and at $\mathfrak{p}_{\infty}^{(3)}$ is +1 .

Of the 113 totally real cubic fields, precisely 55 have an embedding $\mathfrak{p}_{\infty}^{(1)}$ satisfying the condition that $K$ is strictly larger than the Hilbert class field, and for these fields the class number is precisely 3 (so $[K: k]=6$ ), and the corresponding 
Archimedean prime $\mathfrak{p}_{\infty}^{(1)}$ is unique. Since for these cases $G=\operatorname{Gal}(K / k)$ is cyclic of order 6 , the conjectural Stark unit $\epsilon$ would generate the ray class field $K$ over $k$ : Take $\chi: \operatorname{Gal}(K / k) \hookrightarrow \mathbb{C}^{\times}$a faithful character. Then $L^{\prime}(0, \chi) \neq 0$. If $\epsilon^{\sigma^{\prime}}=\epsilon$ for any $\sigma^{\prime} \in \operatorname{Gal}(K / k)$, then

$$
L^{\prime}(0, \chi)=\frac{-1}{2} \sum_{\sigma \sigma^{\prime}} \chi\left(\sigma \sigma^{\prime}\right) \ln \left|\epsilon^{\sigma \sigma^{\prime}}\right|_{w}=\chi\left(\sigma^{\prime}\right) \frac{-1}{2} \sum_{\sigma} \chi(\sigma) \ln \left|\epsilon^{\sigma}\right|_{w}=\chi\left(\sigma^{\prime}\right) L^{\prime}(0, \chi)
$$

so $\chi\left(\sigma^{\prime}\right)=1$ which implies $\sigma^{\prime}=1$ (cf. [26], p. 90). It follows that a numerical confirmation of Stark's Conjecture for these fields should produce explicit algebraic generators for the ray class fields $K$. Given these explicit elements, one can then prove they are indeed units generating the relevant class fields independent of any conjectures used to produce them. These algebraic computations, which are also necessary to complete the numerical confirmation of Stark's Conjecture for these fields, are described in Section 5.

The Archimedean prime $\mathfrak{p}_{\infty}^{(1)}$ is specified by the choice of real root $\beta$ for the polynomial $f(x)$ defining the field $k$, and the ray class of an ideal $\mathfrak{A}$ in the ray class group to conductor $\mathfrak{p}_{\infty}^{(2)} \mathfrak{p}_{\infty}^{(3)}$ is then determined by finding the signs at the two embeddings $\mathfrak{p}_{\infty}^{(2)}$ and $\mathfrak{p}_{\infty}^{(3)}$ of the generator of the principal ideal $\mathfrak{A}^{a}$ where $a$ is the order of the ideal $\mathfrak{A}$ in the usual class group of $k$. For our computations, this data was computed for the relevant prime ideals of $k$ using the Pari-GP calculator, then this information was used to generate the ideals of $k$ of norm less than $N$ in the various ray classes using some symbolic manipulations in Mathematica.

Using this norm data, the values at zero of the derivatives of the partial zeta functions $\zeta^{\prime}(0, \mathfrak{b})$ for each of the six ray classes modulo $\mathfrak{p}_{\infty}^{(2)} \mathfrak{p}_{\infty}^{(3)}$ were computed using the results of the previous section. For most of the examples, the values were computed to a (proved) accuracy of $10^{-35}$, although in some cases more accuracy was required $\left(10^{-70}\right.$ for four of the examples).

This conjecturally computes the six values $\left|\epsilon^{\sigma_{\mathfrak{b}}}\right|=e^{-2 \zeta^{\prime}(0, \mathfrak{b})}$, where the absolute value is defined by a place over $\mathfrak{p}_{\infty}^{(1)}$ of $k$. Since $\epsilon$ in Stark's Conjecture is defined only up to a root of unity, we choose $\epsilon$ by

$$
\epsilon=e^{-2 \zeta^{\prime}\left(0, c^{0}\right)},
$$

where $\mathfrak{c}^{0}$ is the principal ray modulo $\mathfrak{p}_{\infty}^{(2)} \mathfrak{p}_{\infty}^{(3)}$, i.e., so that $\epsilon$ is positive in the embedding lying over $\mathfrak{p}_{\infty}^{(1)}$. The abelian condition in Stark's Conjecture then implies that all the conjugates $\epsilon^{\sigma_{\mathfrak{b}}}$ of $\epsilon$ are positive in this embedding (since $\epsilon^{\sigma_{\mathfrak{b}}-1}$ is a square in $K$, hence is positive, cf. [26], p. 93 and [25]). With this choice of $\epsilon$, we have conjecturally computed the numerical values of the six Galois conjugates

$$
\epsilon^{\sigma_{\mathfrak{b}}}=e^{-2 \zeta^{\prime}(0, \mathfrak{b})}
$$

of $\epsilon$ with respect to the real embedding of $K$ in $\mathbb{R}$ (conjecturally) defined by (4). This embedding defines an Archimedean prime of $K$ lying over $\mathfrak{p}_{\infty}^{(1)}$ and the remaining primes of $K$ over $\mathfrak{p}_{\infty}^{(1)}$ are the conjugates by the Galois group $\operatorname{Gal}(K / k)$ and these are the real primes of $K$. The remaining Archimedean primes of $K$ are complex, lying over $\mathfrak{p}_{\infty}^{(2)}$ and $\mathfrak{p}_{\infty}^{(3)}$ and under the corresponding embeddings of $K$ into $\mathbb{C}$, the element $\epsilon$ and its Galois conjugates should (conjecturally) be nonreal complex numbers of absolute value 1 . 
To determine the algebraic element $\epsilon$, we follow Stark and observe that $\zeta^{\prime}(0, \mathfrak{b})=$ $-\zeta^{\prime}(0, \overline{\mathfrak{b}})$ for every class $\mathfrak{b}$. It follows that the element $\tau$ generating $\operatorname{Gal}(K / H)$ of Section 3 acts by inversion on the conjugates of $\epsilon:\left(\epsilon^{\sigma_{\mathfrak{b}}}\right)^{\tau}=\left(\epsilon^{\sigma_{\mathfrak{b}}}\right)^{-1}$. It follows that the element

$$
A=\epsilon+1 / \epsilon=\operatorname{Tr}_{K / H}(\epsilon)
$$

should be an integral element of the Hilbert class field $H$ (even a generator for $H$ over $k$ ) and that $\epsilon$ satisfies the equation

$$
x^{2}-A x+1=0
$$

over $H$. The conjugates of $A$ under $\operatorname{Gal}(H / k)$ should be the elements $\epsilon^{\sigma_{\mathrm{c}}^{t}}+1 / \epsilon^{\sigma_{\mathrm{c}}^{t}}$ where $\mathfrak{c}$ is a class generating the ray class group and $t=0,1,2$ (the element $A$ corresponds then to $t=0$ ). Hence the element $A$ of $H$ satisfies the cubic equation

$$
x^{3}-s_{1} x^{2}+s_{2} x-s_{3}=0
$$

over $k$, where $s_{i}$ is the $i^{\text {th }}$ elementary symmetric function in the $\operatorname{Gal}(H / k)$ conjugates of $A$ above, and should be an algebraic integer in $k$.

To determine $A$ as an element of $H$ it therefore suffices to determine the algebraic integers

$$
s_{i}=a_{i}+b_{i} \beta+c_{i} \beta^{2}, \quad i=1,2,3,
$$

in (7) in terms of the generator $\beta$ of $k$. In Stark's example in [21], the approximate numerical values of the $s_{i}$ in the embedding defined by $\mathfrak{p}_{\infty}^{(1)}$ were computed assuming the validity of (5). The absolute values of the $s_{i}$ in the remaining two embeddings of $k$ were then bounded using the fact that the Galois conjugates of $\epsilon$ are conjecturally of absolute value 1 in these embeddings. This reduced the determination of $a_{i}, b_{i}, c_{i}$ (which are elements of $\mathbb{Z}$ in Stark's example) to a check of a small number of cases.

We proceeded slightly differently, instead using a standard recognition algorithm to determine the monic polynomials in $\mathbb{Z}[x]$ satisfied by the real number $s_{i}$ in the embedding defined by $\mathfrak{p}_{\infty}^{(1)}$. For most of the cases considered, an accuracy of $10^{-35}$ in the values of the relevant partial zeta functions was sufficient to recognize the necessary monic cubics, but some of the more obstreperous examples required greater accuracy. Once the monic polynomial over $\mathbb{Z}$ satisfied by $s_{i}$ in (8) was determined, the three roots could be determined to arbitrary precision. Equation (8) then gives 3 equations for $a_{i}, b_{i}$ and $c_{i}$ corresponding to the three possible embeddings of $k$ (i.e., corresponding to the three possible choices of roots $\beta_{1}, \beta_{2}$, $\beta_{3}$ of $\left.f(x)\right)$ and the three possible roots of the polynomial satisfied by $s_{i}$. The root $s_{i}$ corresponding to the embedding $\mathfrak{p}_{\infty}^{(1)}$ defined by $\beta_{1}$ is the value used to recognize the polynomial satisfied by $s_{i}$, so in fact there are only two possible orderings of the remaining two roots of this polynomial (which correspond to $\beta_{2}$ and $\beta_{3}$ ) to consider. In all the cases considered, the index of $\mathbb{Z}[\beta]$ in the ring of integers of $k$ was quite small (dividing 6 in fact), so that $a_{i}, b_{i}$ and $c_{i}$ in (8) should be elements of $(1 / 6) \mathbb{Z}$ and hence are easily determined as solutions to one of these two systems of 3 equations by rounding the numerical solutions (in fact it is easy to check that only one of the two possible orderings of roots for $s_{i}$ can produce rational solutions $a_{i}, b_{i}$ and $c_{i}$ ).

Once the coefficients in (8) are determined, it is an easy matter to find the equations over $\mathbb{Q}$ of the elements $A$ and $\epsilon$ defined by equations (8), (7) and (6). For example, the monic polynomial $f_{A}(x)$ in $\mathbb{Z}[x]$ of degree 9 satisfied by $A$ is obtained 
by multiplying together the three cubics $x^{3}-s_{1} x^{2}+s_{2} x-s_{3}$ obtained from (8) by substituting the three values $\beta_{1}, \beta_{2}$, and $\beta_{3}$ to sufficient precision, expanding and rounding the coefficients. Note also that $\epsilon$ is a unit since by construction it satisfies a monic polynomial $f_{\epsilon}(x)$ of degree 18 in $\mathbb{Z}[x]$ with constant term 1 .

In Stark's original cubic example, the Hilbert class field $H$ and ray class field $K$ were known explicitly beforehand and the unit $\epsilon$ was constructed as an element of $K$. In our examples (which include Stark's), the algebraic element $\epsilon$ is not (yet) known to lie in the appropriate ray class field. All that is known at this point is that 6 of 18 roots of a polynomial $f_{\epsilon}(x)$ satisfied by a unit $\epsilon$ in some extension of $k$ are real and agree with the exponentials of the 6 proved approximate values of the partial zeta functions for $k$. The only immediate numerical indication that $\epsilon$ is indeed the required Stark unit is provided by a quick computation of the roots of $f_{\epsilon}(x)$ (to whatever precision one chooses), which shows that the remaining 12 roots are indeed all nonreal complex numbers of absolute value 1. A number of items therefore remain to be proved to complete the numerical confirmation of Stark's conjecture for these examples, which we state as

Theorem 1. Let $k$ be one of the 55 totally real cubic fields of discriminant $D_{k}<$ 50000 with class number divisible by 3 such that the ray class group of conductor $\mathfrak{p}_{\infty}^{(2)} \mathfrak{p}_{\infty}^{(3)}$ for two of the Archimedean primes of $k$ is strictly larger than the Hilbert class field of $k$. Let $\epsilon$ be a root of the polynomial $f_{\epsilon}(x)$ constructed above, let $A=\epsilon+1 / \epsilon$, and set $K=k(\epsilon)$, and $H=k(A)$. Then:

1. The field $H=k(A)$ is the Hilbert class field of $k$.

2. The field $K=k(\epsilon)$ is the ray class field of $k$ of conductor $\mathfrak{p}_{\infty}^{(2)} \mathfrak{p}_{\infty}^{(3)}$.

3. If the embedding of $K$ into $\mathbb{R}$ extending the embedding of $k$ into $\mathbb{R}$ defined by the remaining Archimedean prime $\mathfrak{p}_{\infty}^{(1)}$ of $k$ is fixed by taking $\epsilon$ to the real root of $f_{\epsilon}(x)$ that agrees numerically with the value computed in equation (4), then for each of the remaining five ray classes $\mathfrak{b}$ the image of the Galois conjugate $\sigma_{\mathfrak{b}}(\epsilon)$ agrees numerically with the value $e^{-2 \zeta^{\prime}(0, \mathfrak{b})}$ in (5) (i.e., the Frobenius automorphisms are acting as predicted by Stark's conjecture).

4. The field $K(\sqrt{\epsilon})$ is an abelian extension of $k$.

The proof of Theorem 1 will be given in the following section. Since the computed values of the relevant partial zeta functions have, by the results of Section 3, been proved to be accurate, Theorem 1 immediately gives the following

Corollary. The refined abelian (rank-one) Stark Conjecture is valid for the fields $K / k$ in Theorem 1 to a proved numerical accuracy of at least $10^{-30}$.

The properties in Theorem 1 are essentially completely algebraic in nature and are independent of Stark's conjecture. Note, however, that while they are algebraic we shall in fact use the analytic data given in Stark's conjecture as a 'catalyst' to verify them, in much the same catalytic manner that Stark's conjecture was used to produce them.

\section{Using Stark's Conjecture to COMpute Hilbert and Ray Class fields}

In this section we indicate the proof of the properties of Theorem 1 of the previous section for the 55 cubic examples. In particular this will indicate how Stark's conjecture can be used to provide explicit generators for Hilbert and certain ray class fields. Each of these cubic fields $k$ has class number 3 (and associated ray class number 6 ). 
In each of the computed examples, the monic polynomial of degree 18 satisfied by $\epsilon$ was irreducible over $\mathbb{Q}$. In particular, $[K: k]=6$ and $[H: k]=3$. It follows also that $\epsilon$ in fact generates $K=k(\epsilon)$ over $\mathbb{Q}$, and not just over $k$. Similarly, the element $A$ generates $H=k(A)$ over $\mathbb{Q}$.

The roots of the polynomial $f_{A}(x)$ are all real, so the field $H=k(A)$ is a totally real extension of $k$ of degree 3. Computing the discriminant of $H$ over $\mathbb{Q}$ (using Pari-GP) shows that $D_{H}=D_{k}^{3}$, which implies that $H$ is an unramified extension of $k$. If $H$ were not Galois over $k$, then its Galois closure over $k$ would also be an unramified extension of $k$, of degree 6 , and having the symmetric group $S_{3}$ as Galois group. The quadratic subfield of this extension of $k$ would then be an unramified quadratic extension of $k$, which means that the class number of $k$ would be divisible by 2 , which it is not. It follows that $H$ is in fact Galois over $k$, hence is the Hilbert class field of $k$. This proves (1) of Theorem 1 .

Remark. In computing field discriminants it was necessary in some instances to modify the algebraic integer generator for the field by multiplying by a unit in the field $k$ to produce another integer $\alpha$, the discriminant of whose minimal polynomial, while larger, was nevertheless easier to factor.

We next prove (4) of Theorem 1, which will in particular prove that $K / k$ is an abelian extension. In all examples, the polynomial $f_{\epsilon}\left(x^{2}\right)$ is irreducible over $\mathbb{Q}$, so $[K(\sqrt{\epsilon}): k]=12$ (and $\epsilon$ is never a square in $K$ for these examples). Since $(\sqrt{\epsilon} \pm 1 / \sqrt{\epsilon})^{2}=A \pm 2$, we have

$$
K(\sqrt{\epsilon})=k(\sqrt{A+2}, \sqrt{A-2}) .
$$

Hence $K(\sqrt{\epsilon})$ is an abelian extension of $k$ if (and only if) the extensions $k(\sqrt{A \pm 2})=$ $H(\sqrt{A \pm 2})$ are both abelian extensions of $k$ (of degree 6 , although this is not required). The quadratic extension $H(\sqrt{A \pm 2})$ of $H$ will be Galois over $k$ (hence abelian over $k$, since $H$ is Galois over $k)$ if and only if $\sigma(A \pm 2) /(A \pm 2)$ is a (nonzero) square in $H$ for a generator $\sigma$ of $\operatorname{Gal}(H / k)$. This is equivalent to $\sigma(A \pm 2)(A \pm 2)=-c / \sigma^{2}(A \pm 2)$ being a square in $H$ where $c$ is the constant term of the cubic polynomial satisfied by $A \pm 2$ over $k$, which in turn is equivalent to $-(A \pm 2) / c$ being a square in $H$. Since the minimal polynomial for $A$ was constructed in (7), it is an easy matter to find the minimal polynomial over $k$ for the element $-(A \pm 2) / c$, hence also the minimal polynomial of degree 9 over $\mathbb{Q}$ for this element. In all cases this polynomial factors over $\mathbb{Q}$ into a product of two polynomials of degree 9 when $x^{2}$ is substituted for $x$, and this is a sufficient (and necessary) condition for the element to be a square. This proves (4) of Theorem 1.

To complete the proof of (2) of Theorem 1 it suffices to check that the extension $K / k$ is unramified outside the infinite primes of $k$. Because of the size of the fields involved $([K: \mathbb{Q}]=18)$, it was difficult to compute the discriminant of $K$ over $\mathbb{Q}$ directly (confirming that $D_{K}=D_{k}^{6}$ would prove that $K / k$ was unramified). Instead we determined the unique quadratic extension $F$ of $k$ contained in $K$ and confirmed (by a discriminant calculation on this extension of degree 6 over $\mathbb{Q}$, checking that $D_{F}=D_{k}^{2}$ ) that the extension $F / k$ is also unramified at all finite primes of $k$. To determine the field $F$, observe that $\epsilon$ satisfies the sextic

$$
\left(x^{2}-A x+1\right)\left(x^{2}-\sigma(A) x+1\right)\left(x^{2}-\sigma^{2}(A) x+1\right)
$$

over $k$. Expanding and using the elementary symmetric functions $s_{i}$ of the conjugates of $A$ in equation (7) shows that the minimal polynomial for $\epsilon$ over $k$ is the 
sextic

$$
x^{6}-s_{1} x^{5}+\left(s_{2}+3\right) x^{4}-\left(2 s_{1}+s_{3}\right) x^{3}+\left(s_{2}+3\right) x^{2}-s_{1} x+1 .
$$

The discriminant of this sextic differs by the square of $-s_{1}^{2} s_{2}^{2}+4 s_{2}^{3}+4 s_{1}^{3} s_{3}-$ $18 s_{1} s_{2} s_{3}+27 s_{3}^{2}$ from the element

$$
\Delta=-\left(8+4 s_{1}+2 s_{2}+s_{3}\right)\left(8-4 s_{1}+2 s_{2}-s_{3}\right)
$$

and so $F=k(\sqrt{\Delta})$ (note that the alternating group $A_{6}$ does not contain a cyclic subgroup of order 6 ). This determines a polynomial of degree 6 over $\mathbb{Q}$ defining the field $F$ and allows the determination of the discriminant of $F$, confirming that $F / k$ is unramified and proving that $K$ is the appropriate ray class field.

It remains to prove (3) of Theorem 1 , namely that the numerical values computed in equation (5) of the previous section do in fact correspond to the conjugates by the appropriate Frobenius elements of the algebraic element $\epsilon$. We proceed by using equation (5) to numerically produce an algebraic conjugate of $\epsilon$ and then verify independently that this conjugate is indeed given by the appropriate Frobenius automorphism. Let $\mathfrak{c}$ denote the generator of the ray class group of $k$ of conductor $\mathfrak{p}_{\infty}^{(2)} \mathfrak{p}_{\infty}^{(3)}$ used to compute the values of the partial zeta functions used in equation (5). Suppose $\sigma$ is a generator of $\operatorname{Gal}(K / k)$. Then

$$
\sigma(\epsilon)=a_{0}+a_{1} \epsilon+a_{2} \epsilon^{2}+a_{3} \epsilon^{3}+a_{4} \epsilon^{4}+a_{5} \epsilon^{5}
$$

for some $a_{0}, \ldots, a_{5} \in k$. Conjugating this equation by $\sigma, \sigma^{2}, \ldots, \sigma^{5}$ gives the system of equations

$$
\sigma^{i+1}(\epsilon)=a_{0}+a_{1} \sigma^{i}(\epsilon)+a_{2} \sigma^{i}(\epsilon)^{2}+a_{3} \sigma^{i}(\epsilon)^{3}+a_{4} \sigma^{i}(\epsilon)^{4}+a_{5} \sigma^{i}(\epsilon)^{5}
$$

for $i=0,1, . ., 5\left(\sigma^{6}=1\right)$. Assume for the moment that Stark's conjecture in equation (5) is valid and take $\sigma=\sigma_{\mathfrak{c}}$, the Frobenius element for the ray class $\mathfrak{c}$ in $\operatorname{Gal}(K / k)$. Then we have the numerical values of the elements in the system of 6 equations in (12), from which we can solve numerically for the six coefficients $a_{0}, \ldots, a_{5}$. We then recognize the cubic polynomials satisfied by these elements and use these to determine the elements $a_{0}, \ldots, a_{5}$ as polynomials with $\mathbb{Q}$-coefficients in the elements $1, \beta, \beta^{2}$.

Remark. As a computational matter, the system of numerical equations (12) is rewritten in order to write each $a_{i}$ as the quotient of two algebraic integers in $k$ since this provides an easy check on the recognition algorithm (namely, whether the resulting cubic polynomial is monic). Also, since the polynomial $f_{\epsilon}(x) \in \mathbb{Z}[x]$ has been determined, we can, under the assumption of the validity of Stark's conjecture, determine the numerical values in equation (5) to arbitrary precision by solving for the appropriate root of $f_{\epsilon}(x)$. In the computations of the Frobenius automorphism above, typically 500 digits of accuracy were required.

We now use these exact elements $a_{0}, \ldots, a_{5}$ of $k$ to define $\sigma(\epsilon)$ by equation (11). It is then immediate to verify that the resulting $\sigma(\epsilon)$ is again a root of the minimal polynomial of $\epsilon$ over $k$ in equation (9), so that the map $\epsilon \mapsto \sigma(\epsilon)$ defines an element of $\operatorname{Gal}(K / k)$, and that this automorphism is of order 6 . This proves that there is an algebraic automorphism $\sigma \in \operatorname{Gal}(K / k)$ such that the numerical values of the conjugates of $\epsilon$ agree with the values on the right hand side of equation (5). To complete the proof of Theorem 1 it therefore suffices to show that this automorphism $\sigma$ is the Frobenius automorphism corresponding to the class $\mathfrak{c}$ in the extension $K / k$ (and 
not its inverse, the only other possibility in this case). Let $\mathfrak{p}$ (dividing the prime $p$ in $\mathbb{Z}$ ) be a prime of $k$ of absolute degree 1 lying in the class $\mathfrak{c}$ (a list of such primes is produced in the original computation of the partial zeta function values), such that $\mathfrak{p}$ does not divide the discriminant of the polynomial $f_{\epsilon}(x)$. If $\mathcal{O}_{K}$ denotes the ring of integers of $K$, then $\mathcal{O}_{K}=\mathbb{Z}[\epsilon]+\mathfrak{P}$ and $\mathcal{O}_{K} / \mathfrak{P}=\mathbb{Z} / p \mathbb{Z}$ for any prime $\mathfrak{P}$ of $K$ dividing $\mathfrak{p}$ (note that $\mathfrak{p}$ splits completely in $K$ ). It follows that to confirm that $\sigma=\sigma_{\mathfrak{c}}$ it suffices to verify that

$$
\sigma(\epsilon) \equiv \epsilon^{p} \quad \bmod p
$$

for the $\sigma(\epsilon)$ defined by equation (11). This is an elementary computation, since $\beta \equiv b \bmod \mathfrak{P}$ for a (known) rational integer $b$, so that $\sigma(\epsilon) \in \mathbb{Z}[\epsilon] \bmod \mathfrak{P}$ and equation (13) becomes an equality of two polynomials in $\mathbb{Z}[x]$ in the quotient ring $\mathbb{Z}[x] /\left(p, f_{\epsilon}(x)\right)$. The confirmation that these equations are satisfied for all the examples completes the proof of Theorem 1.

In general it is of interest to know whether the element $\epsilon$ in Stark's conjecture can be taken to be a square in $K$ (for example, cf. [3]), and to examine the ramification properties of the extension $K(\sqrt{\epsilon}) / k$ (cf. [27] ). As mentioned in the proof of Theorem 1 , none of the polynomials $f_{\epsilon}\left(x^{2}\right)$ factors over $\mathbb{Q}$, and one similarly finds that also $f_{\epsilon}\left(-x^{2}\right)$ remains irreducible. It follows that none of the elements $\pm \epsilon$ are squares in $K$ for these examples. Also, since none of these fields has a totally positive system of fundamental units, the ray class field to conductor $\mathfrak{p}_{\infty}^{(1)} \mathfrak{p}_{\infty}^{(2)} \mathfrak{p}_{\infty}^{(3)}$ (the strict Hilbert class field) is the same as the field $K$. It follows that the quadratic extensions $K(\sqrt{ \pm \epsilon})$ over $K$ ramify at some prime above 2 . We did not determine whether a particular choice of sign minimizes the different of the extension.

Corollary. For the examples in Theorem 1, the elements $\pm \epsilon$ are not squares in $K$ and the two quadratic extensions $K(\sqrt{ \pm \epsilon})$ are both ramified at some prime above 2.

It is not clear whether the fact that none of the Stark units for these 55 examples were squares in the corresponding ray class field $K$ is significant (although in light of [3] it is suggestive). The group of units of $K$ modulo squares of units is an elementary abelian 2-group of rank 12 , so that the probability that a randomly chosen unit of $K$ is a square is $1 / 2^{12}$. The probability that of 110 randomly chosen units none of them is a square is then approximately 0.9735 , so this sample size is too small to be predictive.

\section{EXAMPLE: DISCRIMINANT 28212}

As previously mentioned, in Stark's original cubic example the Hilbert class field $H$ is obtained over $k$ by composing with an abelian extension of $\mathbb{Q}$ (namely $\mathbb{Q}(\cos (2 \pi) / 7))$ and our numerical investigations originated in trying to construct a similar but more generic cubic example in which $H$ is not a 'genus class field'. The first example of such a field occurs for a discriminant $D_{k}=28212$. In this section we indicate some of the details of the computations described in the previous sections for this cubic field, in particular finding explicit generators for the Hilbert class field and using this to find the Galois closure of $H$ and prove that $H$ is not obtained by composing any cubic extension of $\mathbb{Q}$ (let alone abelian) with $k$. The example described here also serves as a paradigm for the abbreviated data for the other examples in the tables in Section 7.

There are 3 nonisomorphic totally real cubic fields of discriminant $28212=2^{2}$. $3 \cdot 2351$, defined by the polynomials $x^{3}-x^{2}-37 x-47, x^{3}-x^{2}-41 x+93$, and 
$x^{3}-x^{2}-53 x+153$. Up to isomorphism there is precisely one totally real cubic field of discriminant $7053=3 \cdot 2351$, defined by the polynomial $x^{3}-x^{2}-23 x+48$.

The field $k=\mathbb{Q}(\beta)$ with $\beta^{3}-\beta^{2}-41 \beta+93=0$ satisfies the conditions at the beginning of Section 4: $k$ has class number divisible by 3 (in fact equal to 3), and if $\mathfrak{p}_{\infty}^{(1)}$ is the Archimedean prime defined by the root $\beta=5.40269 \ldots$, then the ray class field $K$ of conductor $\mathfrak{p}_{\infty}^{(2)} \mathfrak{p}_{\infty}^{(3)}$ is strictly larger than the Hilbert class field. The ray class $\mathfrak{c}$ containing the prime $\mathfrak{p}=(5, \beta-2)$ generates the corresponding ray class group. [The field defined by the equation $x^{3}-x^{2}-53 x+153$ also satisfies the necessary hypotheses of Section 4 . The corresponding data for this field appears in the tables in Section 7.]

Using an internal accuracy of 150 digits and computing 350 residues to insure an accuracy of at least $10^{-55}$, one finds

$\zeta^{\prime}\left(0, \mathfrak{c}^{0}\right)=4.62040289671991440543776590019223277624303967323957791671782 \ldots$

$\zeta^{\prime}\left(0, \mathfrak{c}^{1}\right)=-5.23781296974710843037213422039206497492945318572477673266825 \ldots$

$\zeta^{\prime}\left(0, \mathfrak{c}^{2}\right)=2.259302064318993328125265486811196491695081900397339500930079 \ldots$

yielding potential Stark units

$$
\begin{aligned}
& \epsilon_{1}=10309.34241235303439168049341491290535905308263827742948201871 \ldots \\
& \epsilon_{2}=0.000028215870927227640203451517278794044636382871473311203053 \ldots \\
& \epsilon_{3}=91.70749672416162340728363966075410917807739071597377629976299 \ldots
\end{aligned}
$$

The numerical values of the traces $(\epsilon+1 / \epsilon)$ of these units are then

$$
\begin{aligned}
& A_{1}=10309.34250935243163759630564450029748610493408547697070477070 \ldots \\
& A_{2}=35441.04675610630550032056420255885270767586064066439842279032 \ldots \\
& A_{3}=91.71840095811981121163526964493461769139799224062807444575130 \ldots
\end{aligned}
$$

the elementary symmetric functions of which are:

$$
\begin{aligned}
& s_{1}=45842.10766641685694912850511670408481147219271838199720200678 \ldots \\
& s_{2}=3.695700424453132417259675515402850795764644990380044622513860 \ldots \\
& s_{3}=3.351150893335448884121299970610876103784771962239631343594939 \ldots
\end{aligned}
$$

Using a standard recognition algorithm, one finds that these real numbers are roots of the following cubic polynomials:

$$
\begin{aligned}
& p_{1}(x)=-238095-234141 x-45837 x^{2}+x^{3}, \\
& p_{2}(x)=3826481704-164574444 x-369570042 x^{2}+x^{3}, \\
& p_{3}(x)=-132604621897+189189597635 x-33511508939 x^{2}+x^{3} .
\end{aligned}
$$

Using these polynomials one finds

$$
\begin{aligned}
& s_{1}=-22071+5644 \beta+1282 \beta^{2}, \\
& s_{2}=-177889957+45498408 \beta+10334235 \beta^{2}, \\
& s_{3}=-16130530946+4125659898 \beta+937077613 \beta^{2} .
\end{aligned}
$$

Using these exact values we can now find the $9^{\text {th }}$ degree equation satisfied by $A$ : Take the cubic $x^{3}-s_{1} x^{2}+s_{2} x-s_{3}$, substitute the 3 possible conjugates of $\beta$ 
(computed numerically to high precision), multiply these cubics together and round the (integer) coefficients. The result is the polynomial

$$
\begin{aligned}
f_{A}(x)= & x^{9}-45837 x^{8}+369335901 x^{7}-31624086134 x^{6}-169410056472 x^{5} \\
& -164382128003 x^{4}+465586425823 x^{3}+980522077951 x^{2} \\
& +392287249570 x-132604621897 .
\end{aligned}
$$

Applying "initalgred" in Pari-GP to this polynomial $f(x)$ to find another field generator for $H=k(A)$ gives the polynomial

$$
x^{9}-x^{8}-25 x^{7}+2 x^{6}+190 x^{5}+127 x^{4}-307 x^{3}-167 x^{2}+150 x-23
$$

with field discriminant $D_{H}=22454408824128=2{ }^{6} 3^{3} 2351^{3}=D_{k}^{3}$, proving as in Section 5 that $H$ is the Hilbert class field for $k$.

The polynomial of degree 18 satisfied by $\epsilon$ is determined by taking the product of $x^{2}-A x+1$ over all the 9 roots of $f_{A}(x)$ and gives

$$
\begin{aligned}
f_{\epsilon}(x)= & x^{18}-45837 x^{17}+369335910 x^{16}-31624452830 x^{15}-166824705129 x^{14} \\
& -354127928243 x^{13}-373707802532 x^{12}-151370292943 x^{11} \\
& +107872718980 x^{10}+209661834717 x^{9}+107872718980 x^{8} \\
& -151370292943 x^{7}-373707802532 x^{6}-354127928243 x^{5} \\
& -166824705129 x^{4}-31624452830 x^{3}+369335910 x^{2}-45837 x+1 .
\end{aligned}
$$

The absolute values of the roots of $f_{\epsilon}(x)$ are given approximately by

$$
\begin{aligned}
& \{1 ., 1 ., 1 ., 1 ., 1 ., 1 ., 1 ., 1 ., 1 ., 1 ., 1 ., 1 ., 35441.04672789043,0.0000969993972459158, \\
& \text { 0.0109042339581878, 10309.34241235303, 0.00002821587092722764, }
\end{aligned}
$$

$91.7074967241616\}$.

The computed values of $\epsilon_{1}, \epsilon_{2}$, and $\epsilon_{3}$ given by Stark's conjecture from the values of the partial zeta functions differ from the last three roots of $f_{\epsilon}(x)$ above by approximately $10^{-60}, 10^{-68}$, and $10^{-61}$, respectively.

The generator $\sigma_{\mathfrak{c}}$ of $\operatorname{Gal}(K / k) \cong \mathbb{Z} / 6 \mathbb{Z}$ (determined as described in Section 5 and verified by confirming that $\sigma_{\mathfrak{c}}(\epsilon) \equiv \epsilon^{5} \bmod \mathfrak{p}$ with $\left.\mathfrak{p}=(5, \beta-2)\right)$ is given explicitly by

$$
\sigma_{\mathfrak{c}}(\epsilon)=\left(a_{0}+a_{1} \epsilon+a_{2} \epsilon^{2}+a_{3} \epsilon^{3}+a_{4} \epsilon^{4}+a_{5} \epsilon^{5}\right) / 35626819129793805949
$$

with

$$
\begin{aligned}
a_{0}= & -253021406556793673085768+63919617157029310621856 \beta \\
& +14582888259664145092184 \beta^{2}, \\
a_{1}= & 2577370712090340691346989569-659208900700762693152742032 \beta \\
& -149728575434905137542429385 \beta^{2},
\end{aligned}
$$




$$
\begin{aligned}
a_{2}=- & 239408540778937420780089655905+61232837117469668680252067562 \beta \\
& +13908059107414446684962003979 \beta^{2}, \\
a_{3}=2640222158834310099968203077-675284692296064477088106958 \beta & \\
& -153379895000330184633573814 \beta^{2}, \\
a_{4}=- & 337418618277039567474827+84163296584968335168506 \beta \\
& +19291389389815096078965 \beta^{2} \\
a_{5}=- & 3599270210410146021309+144456675064152741936 \beta \\
& +96062588091859333029 \beta^{2} .
\end{aligned}
$$

The Hilbert class field $H$ here has an interesting Galois closure, which in particular proves that $H$ is not obtained by composing any cubic extension of $\mathbb{Q}$ with $k$ (so $H$ is certainly not a genus field for $k$ ).

The Galois closure of $k=\mathbb{Q}(\beta)$ is the field $\mathbb{Q}(\beta, \sqrt{7053})$ whose class number is 9 , with class group isomorphic to $\mathbb{Z} / 3 \mathbb{Z} \times \mathbb{Z} / 3 \mathbb{Z}$. Since the polynomial $x^{3}-x^{2}-23 x+48$ mentioned previously has discriminant 7053 and defines a totally real cubic field, it is easy to see that the Hilbert class field $L$ to $\mathbb{Q}(\beta, \sqrt{7053})$ is given by $L=$ $H(\alpha, \sqrt{7053})$. The field $L$ is a Galois extension of $\mathbb{Q}$ since $\mathbb{Q}(\beta, \sqrt{7053})$ is Galois over $\mathbb{Q}$. The field diagram is the following:

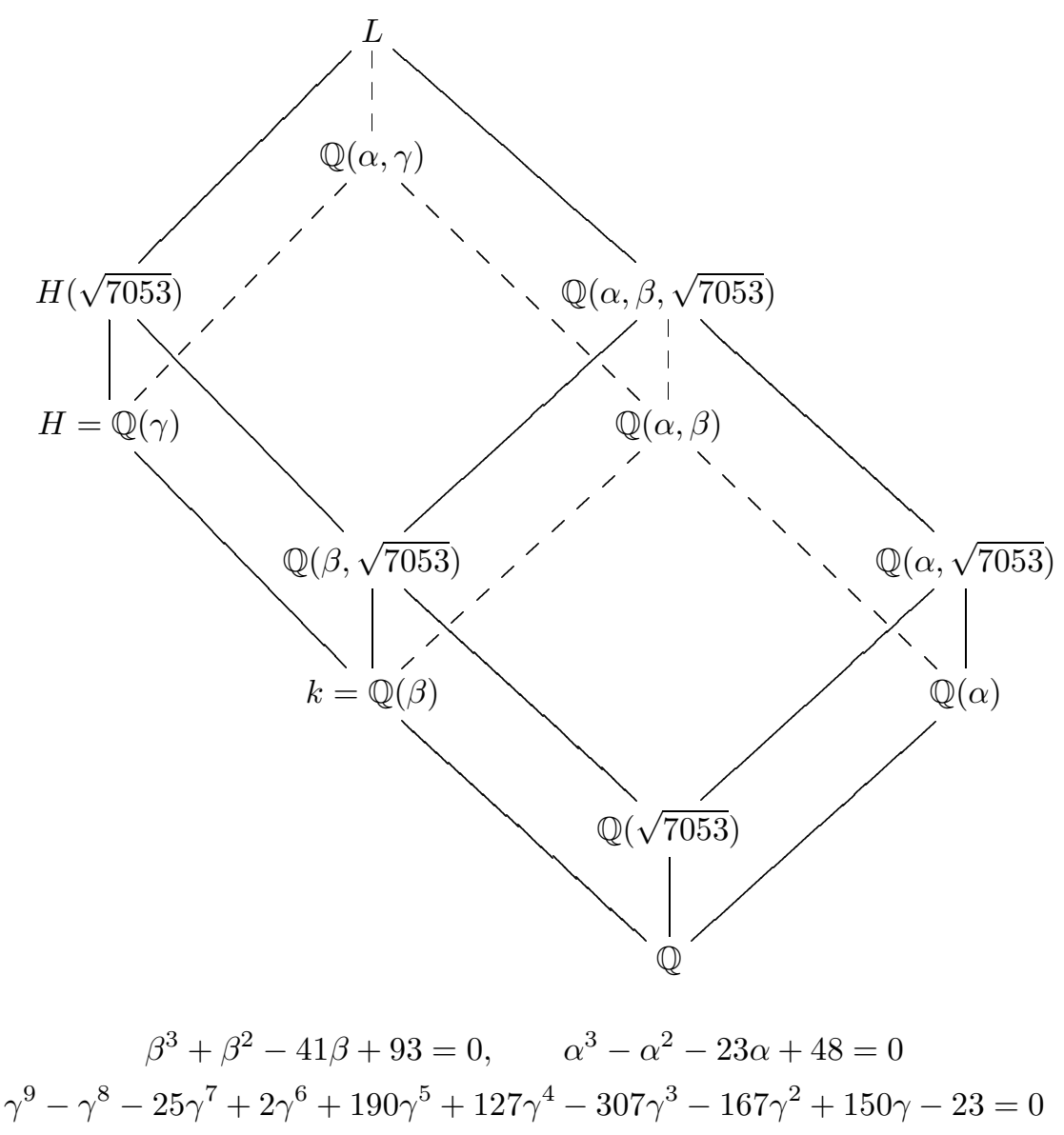


The subgroup $\operatorname{Gal}(L / \mathbb{Q}(\alpha, \sqrt{7053}))$ is a group of order 9. Analyzing the splitting of the prime 2 in $L / \mathbb{Q}$ shows that $\operatorname{Gal}(L / \mathbb{Q}(\alpha, \sqrt{7053})$ ) cannot be cyclic (otherwise the extension $\mathbb{Q}(\alpha, \beta, \sqrt{7053}) / \mathbb{Q}(\alpha, \sqrt{7053})$ would be unramified at 2 , which it is not). The prime 31 splits in $H(\sqrt{7053})$ into 6 primes of degree 1 and 4 primes of degree 3 , so this extension is not Galois over $\mathbb{Q}$ and the subgroup $\operatorname{Gal}(L / H(\sqrt{7053}))$ is a nonnormal subgroup of $\operatorname{Gal}(L / \mathbb{Q})$ of order 3. It is relatively straightforward from this to determine the structure of $\operatorname{Gal}(L / \mathbb{Q})$. Let $\langle\sigma\rangle=\operatorname{Gal}(L / H(\sqrt{7053})),\langle\tau\rangle=$ $\operatorname{Gal}(L / \mathbb{Q}(\alpha, \beta, \sqrt{7053})),\langle\tau, \rho\rangle=\operatorname{Gal}(L / \mathbb{Q}(\alpha, \sqrt{7053}))$, and $\langle\pi\rangle=\operatorname{Gal}(L / H(\alpha))$. Then $\operatorname{Gal}(L / \mathbb{Q})$ is a group of order 54 , the semidirect product of the unique nonabelian group of order 27 in which every element has order 3 by a subgroup of order 2 :

$$
G=\langle\sigma, \tau, \rho\rangle \rtimes\langle\pi\rangle
$$

with $\pi$ inverting $\sigma$ and $\rho$, and centralizing $\tau$ (which generates the center of $G$ ).

It follows easily that the field $H=\mathbb{Q}(\gamma)$ contains a unique cubic subfield, namely $k=\mathbb{Q}(\beta)$. In particular, $H$ is not obtained by composing $k$ with any other cubic extension of $\mathbb{Q}$.

It is interesting to note that the extension $\mathbb{Q}(\alpha, \beta)$ is a non-Galois extension of $\mathbb{Q}$ of degree 9 containing 4 (non-Galois) cubic subfields (the maximum possible). In fact the four subfields are precisely the 3 (up to isomorphism) totally real cubic fields of discriminant 28212 and the unique (up to isomorphism) totally real cubic field of discriminant 7053 mentioned at the beginning of this section. The field $L$ contains 12 cubic subfields (the Galois conjugates of these 4 cubic fields).

\section{TABLES}

The tables below give a complete list of all 55 totally real cubic fields of discriminant less than 50000 with class number divisible by 3 for which there is an abelian extension unramified at all finite primes strictly larger than the Hilbert class field, as in the statement of Theorem 1 in Section 4 (note there are a total of 113 such totally real cubic fields without the condition on the ray class field). These fields all have class number 3 , and for each we give the following abbreviated data:

1. The discriminant $D_{k}$.

2. A cubic polynomial $f(x)$ defining the field $k$.

3. A root $\beta$ of $f(x)$ defining the Archimedean prime $\mathfrak{p}_{\infty}^{(1)}$.

4. A prime $\mathfrak{p}$ of degree 1 in $k$ whose class $\mathfrak{c}$ generates the ray class group of conductor $\mathfrak{p}_{\infty}^{(2)} \mathfrak{p}_{\infty}^{(3)}$ of $k$ and with respect to which the partial zeta function values were computed.

5. The first few digits of the computed Stark units $\epsilon_{\mathfrak{c}^{0}}, \epsilon_{\mathfrak{c}^{1}}$, and $\epsilon_{\mathfrak{c}^{2}}$ in equation (5) of Section 4 , corresponding to the classes $\mathfrak{c}^{0}, \mathfrak{c}^{1}$, and $\mathfrak{c}^{2}$, respectively.

6. The elementary symmetric functions $s_{1}, s_{2}$, and $s_{3}$ of equation (7) in Section 4 as elements in the field $k=\mathbb{Q}(\beta)$.

As described in the example in Section 5 , this data is enough to easily reconstruct most of the information required to numerically confirm Stark's Conjecture for these fields:

(a) The reciprocals of the elements in item 5 above are the computed Stark units corresponding to the classes $\mathfrak{c}^{3}, \mathfrak{c}^{4}$, and $\mathfrak{c}^{5}$, respectively. The computed values of the derivatives at 0 of the partial zeta functions are $(-1 / 2)$ times the logs of the corresponding Stark unit values as in equation (5) of Section 4. 
(b) The polynomials $f_{A}(x)$ and $f_{\epsilon}(x)$ in Section 4 are computed from equations (6) and (7). Alternatively, the sextic equation satisfied by $\epsilon$ over $k$ is given by equation (9) in Section 5 . The polynomial $f_{A}(x)$ defines the Hilbert class field for $k$. The polynomial $f_{\epsilon}(x)$ defines the ray class field $K$ of conductor $\mathfrak{p}_{\infty}^{(2)} \mathfrak{p}_{\infty}^{(3)}$ of $k$ and six of the roots agree to at least $10^{-30}$ with the numerical values of the Stark units.

(c) The quadratic subfield $F=k(\sqrt{\Delta})$ of $K$ containing $k$ is given by equation (10) in Section 5.

$$
\begin{gathered}
D_{k}=2597, \quad f(x)=x^{3}-x^{2}-9 x+8 \quad \beta=3.07911886 \ldots, \quad \mathfrak{p}=(2, \beta) \\
\epsilon_{\mathfrak{c}^{0}}=0.0725901617213239 \ldots, \quad \epsilon_{\mathfrak{c}^{1}}=0.5730726554327768 \ldots, \\
\epsilon_{\mathfrak{c}^{2}}=2.0682046655250200 \ldots, \\
s_{1}=\beta^{2}+3 \beta, \quad s_{2}=5 \beta^{2}+12 \beta-11, \quad s_{3}=6 \beta^{2}+13 \beta-15
\end{gathered}
$$

$$
\begin{aligned}
& D_{k}=4212, \quad f(x)=x^{3}-12 x+10, \quad \beta=2.93045374 \ldots, \quad \mathfrak{p}=(7, \beta+1) \\
& \epsilon_{\mathfrak{c}^{0}}=0.0276040307201833 \ldots, \quad \epsilon_{\mathfrak{c}^{1}}=2.7719617903976693 \ldots, \\
& \epsilon_{\mathfrak{c}^{2}}=0.2889417781460776 \ldots, \\
& s_{1}=3 \beta^{2}+9 \beta-9, \quad s_{2}=19 \beta^{2}+56 \beta-66, \quad s_{3}=31 \beta^{2}+91 \beta-107
\end{aligned}
$$

$$
\begin{gathered}
D_{k}=6885, \quad f(x)=x^{3}-12 x+1, \quad \beta=-3.50503972 \ldots, \quad \mathfrak{p}=(2, \beta+1) \\
\epsilon_{\mathfrak{c}^{0}}=0.0122447097951284 \ldots, \quad \epsilon_{\mathfrak{c}^{1}}=0.6827644272956277 \ldots,
\end{gathered}
$$$$
\epsilon_{\mathfrak{c}^{2}}=2.1227821547518977 \ldots \text {, }
$$$$
s_{1}=3 \beta^{2}-13 \beta+4, \quad s_{2}=16 \beta^{2}-54 \beta+7, \quad s_{3}=18 \beta^{2}-65 \beta+6
$$

$$
\begin{aligned}
& D_{k}=9653, \quad f(x)=x^{3}-14 x+7, \quad \beta=-3.97027720 \ldots, \quad \mathfrak{p}=(2, \beta+1) \\
& \epsilon_{\mathfrak{c}^{0}}=0.0059776944016633 \ldots, \quad \epsilon_{\mathfrak{c}^{1}}=1.6671321960319223 \ldots, \\
& \epsilon_{\mathfrak{c}^{2}}=2.1686999050750842 \ldots, \\
& s_{1}=5 \beta^{2}-21 \beta+10, \quad s_{2}=25 \beta^{2}-98 \beta+42, \quad s_{3}=30 \beta^{2}-119 \beta+52
\end{aligned}
$$

$$
\begin{gathered}
D_{k}=9800, \quad f(x)=x^{3}-x^{2}-23 x-13, \quad \beta=-0.58920487 \ldots, \quad \mathfrak{p}=(3, \beta+2) \\
\epsilon_{\mathfrak{c}^{0}}=0.1812901941037724 \ldots, \quad \epsilon_{\mathfrak{c}^{1}}=0.5401769290063933 \ldots, \\
\epsilon_{\mathfrak{c}^{2}}=0.0115322865834235 \ldots, \\
s_{1}=-9 / 2 \beta^{2}+7 \beta+201 / 2, \quad s_{2}=-69 / 2 \beta^{2}+55 \beta+1519 / 2, \\
s_{3}=-57 \beta^{2}+91 \beta+1255
\end{gathered}
$$

$$
\begin{aligned}
& D_{k}=10309, \quad f(x)=x^{3}-x^{2}-17 x-14, \quad \beta=-3.06021983 \ldots, \quad \mathfrak{p}=(7, \beta) \\
& \epsilon_{\mathfrak{c}^{0}}=0.0025511978534124 \ldots, \quad \epsilon_{\mathfrak{c}^{1}}=10.3857705742642579 \ldots, \\
& \epsilon_{\mathfrak{c}^{2}}=0.1673551471759631 \ldots, \\
& s_{1}=24 \beta^{2}-97 \beta-113, \quad s_{2}=382 \beta^{2}-1552 \beta-1746 \\
& s_{3}=1466 \beta^{2}-5952 \beta-6705
\end{aligned}
$$

$$
\begin{gathered}
D_{k}=11417, \quad f(x)=x^{3}-x^{2}-30 x+71, \quad \beta=4.09084660 \ldots, \quad \mathfrak{p}=(11, \beta+1) \\
\epsilon_{\mathfrak{c}^{0}}=0.0023925102587428 \ldots, \quad \epsilon_{\mathfrak{c}^{1}}=1.6243927034232794 \ldots, \\
\epsilon_{\mathfrak{c}^{2}}=0.3345168756484259 \ldots, \\
s_{1}=35 \beta^{2}+108 \beta-604, \quad s_{2}=194 \beta^{2}+599 \beta-3364 \\
s_{3}=259 \beta^{2}+800 \beta-4495
\end{gathered}
$$




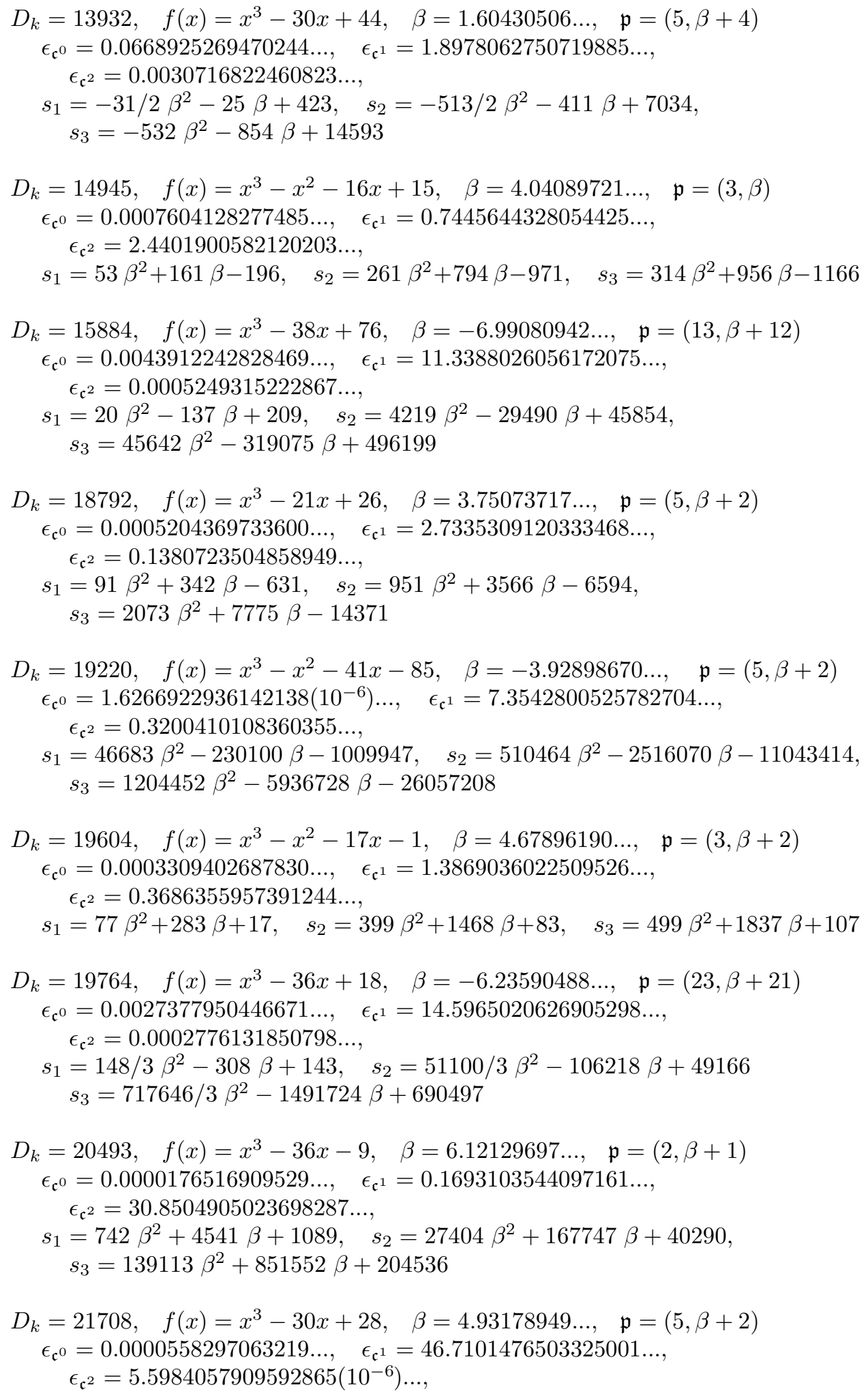


1260 DAVID S. DUMMIT, JONATHAN W. SANDS, AND BRETT A. TANGEDAL

$$
\begin{aligned}
& s_{1}=4575 \beta^{2}+22563 \beta-25971, \quad s_{2}=74674731 \beta^{2}+368280054 \beta-423962226, \\
& s_{3}=3479677527 \beta^{2}+17161037073 \beta-19755703453 \\
& D_{k}=21805, \quad f(x)=x^{3}-x^{2}-30 x-20, \quad \beta=-0.69384876 \ldots, \quad \mathfrak{p}=(31, \beta+17) \\
& \epsilon_{\mathfrak{c}^{0}}=0.4621517145187437 \ldots, \quad \epsilon_{\mathfrak{c}^{1}}=0.4569752565642212 \ldots, \\
& \epsilon_{\mathfrak{c}^{2}}=0.0011044873232772 \ldots \\
& s_{1}=-67 / 2 \beta^{2}+113 / 2 \beta+966, \quad s_{2}=-176 \beta^{2}+298 \beta+5071 \text {, } \\
& s_{3}=-463 / 2 \beta^{2}+785 / 2 \beta+6673 \\
& D_{k}=25137, \quad f(x)=x^{3}-21 x+21, \quad \beta=-5.01842411 \ldots, \quad \mathfrak{p}=(5, \beta+2) \\
& \epsilon_{\mathfrak{c}^{0}}=0.0001276177357691 \ldots, \quad \epsilon_{\mathfrak{c}^{1}}=5.5094459367718433 \ldots, \\
& \epsilon_{\mathfrak{c}^{2}}=0.5976546993579983 \ldots \\
& s_{1}=144 \beta^{2}-721 \beta+599, \quad s_{2}=1144 \beta^{2}-5740 \beta+4784, \\
& s_{3}=1856 \beta^{2}-9316 \beta+7772 \\
& D_{k}=26568, \quad f(x)=x^{3}-30 x+8, \quad \beta=0.26730330 \ldots, \quad \mathfrak{p}=(59, \beta+49) \\
& \epsilon_{\mathfrak{c}^{0}}=0.2230517161304252 \ldots, \quad \epsilon_{\mathfrak{c}^{1}}=424.2073428894345372 \ldots, \\
& \epsilon_{\mathfrak{c}^{2}}=0.0006441139261552 \ldots \text {, } \\
& s_{1}=-133 / 2 \beta^{2}-18 \beta+1991, \quad s_{2}=-44847 / 2 \beta^{2}-5994 \beta+671102 \text {, } \\
& s_{3}=-104062 \beta^{2}-27816 \beta+3114425 \\
& D_{k}=27297, \quad f(x)=x^{3}-21 x+19, \quad \beta=4.03644491 \ldots, \quad \mathfrak{p}=(13, \beta+1) \\
& \epsilon_{\mathfrak{c}^{0}}=0.0000448038334809 \ldots, \quad \epsilon_{\mathfrak{c}^{1}}=10.6678617703943195 \ldots, \\
& \epsilon_{\mathfrak{c}^{2}}=0.1661315669787857 \ldots \text {, } \\
& s_{1}=801 \beta^{2}+3234 \beta-3768, \quad s_{2}=13570 \beta^{2}+54775 \beta-63874 \text {, } \\
& s_{3}=53292 \beta^{2}+215110 \beta-250852 \\
& D_{k}=28212, \quad f(x)=x^{3}-x^{2}-41 x+93, \quad \beta=5.40268750 \ldots, \quad \mathfrak{p}=(5, \beta+3) \\
& \epsilon_{\mathfrak{c}^{0}}=0.0000969993972459 \ldots, \quad \epsilon_{\mathfrak{c}^{1}}=91.7074967241616234 \ldots, \\
& \epsilon_{\mathfrak{c}^{2}}=0.0000282158709272 \ldots \text {, } \\
& s_{1}=1282 \beta^{2}+5644 \beta-22071, \quad s_{2}=10334235 \beta^{2}+45498408 \beta-177889957 \text {, } \\
& s_{3}=937077613 \beta^{2}+4125659898 \beta-16130530946 \\
& D_{k}=28212, \quad f(x)=x^{3}-x^{2}-53 x+153, \quad \beta=-8.00644491 \ldots, \quad \mathfrak{p}=(59, \beta+51) \\
& \epsilon_{\mathfrak{c}^{0}}=0.0046032173748599 \ldots, \quad \epsilon_{\mathfrak{c}^{1}}=43.8735851311032181 \ldots, \\
& \epsilon_{\mathfrak{c}^{2}}=0.0000977972023669 \ldots, \\
& s_{1}=135 / 2 \beta^{2}-608 \beta+2583 / 2, \quad s_{2}=17253 \beta^{2}-155387 \beta+329690, \\
& s_{3}=1255585 / 2 \beta^{2}-5654176 \beta+23993705 / 2 \\
& D_{k}=28392, \quad f(x)=x^{3}-x^{2}-43 x+103, \quad \beta=2.67353762 \ldots, \quad \mathfrak{p}=(3, \beta+2) \\
& \epsilon_{\mathfrak{c}^{0}}=0.2930997997303122 \ldots, \quad \epsilon_{\mathfrak{c}^{1}}=824.5691966980162811 \ldots, \\
& \epsilon_{\mathfrak{c}^{2}}=0.0613118204336775 \ldots \text {, } \\
& s_{1}=-63 / 2 \beta^{2}-53 \beta+2423 / 2, \quad s_{2}=-1235 / 2 \beta^{2}-1033 \beta+47581 / 2 \text {, } \\
& s_{3}=-1859 \beta^{2}-3111 \beta+71619 \\
& D_{k}=29204, \quad f(x)=x^{3}-x^{2}-37 x-69, \quad \beta=7.33096819 \ldots, \quad \mathfrak{p}=(23, \beta) \\
& \epsilon_{\mathfrak{c}^{0}}=0.0000648735003401 \ldots, \quad \epsilon_{\mathfrak{c}^{1}}=2.3361879218197433 \ldots, \\
& \epsilon_{\mathfrak{c}^{2}}=0.0263672975859719 \ldots \\
& s_{1}=141 \beta^{2}+893 \beta+1331, \quad s_{2}=5729 \beta^{2}+36272 \beta+53929,
\end{aligned}
$$




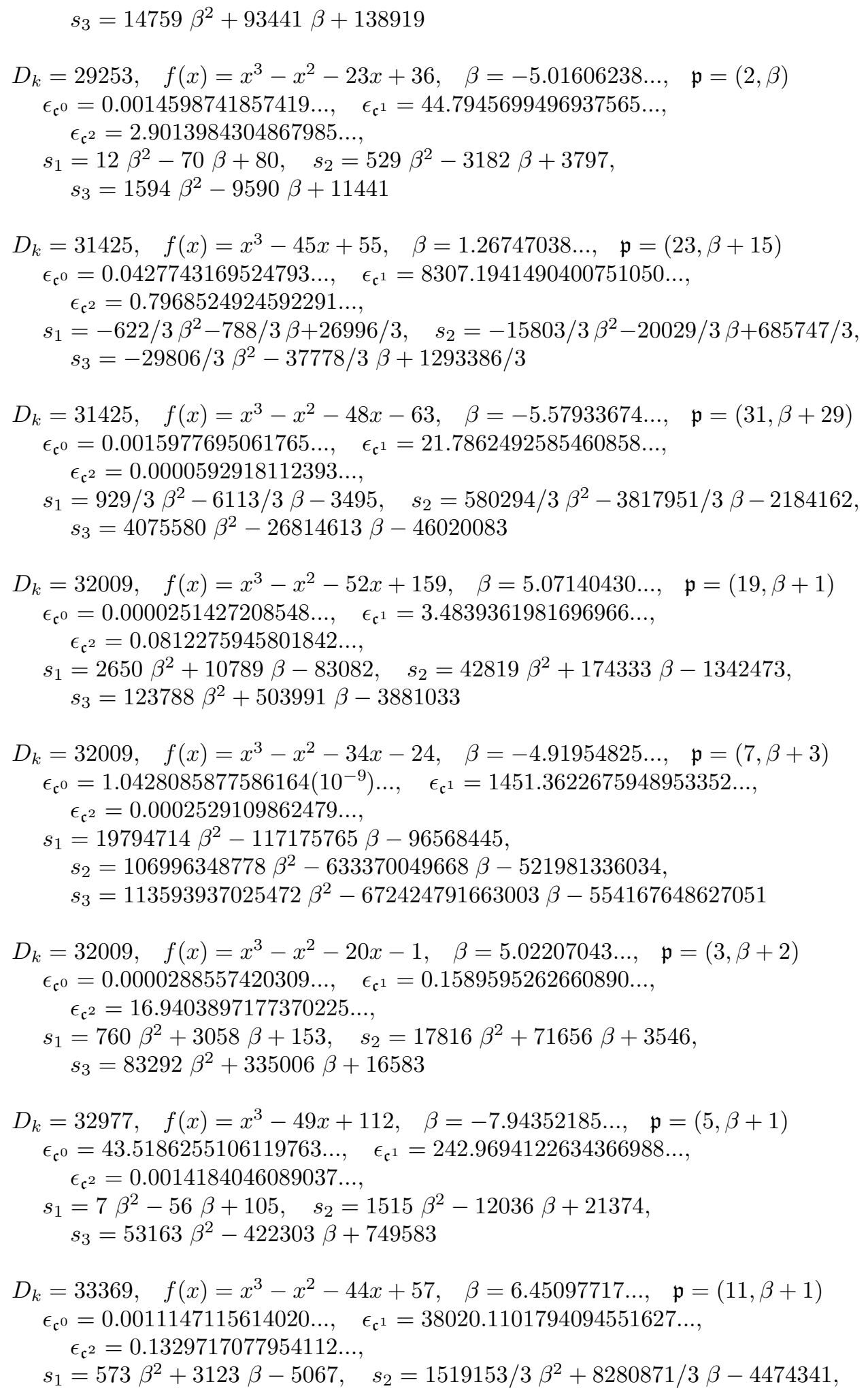




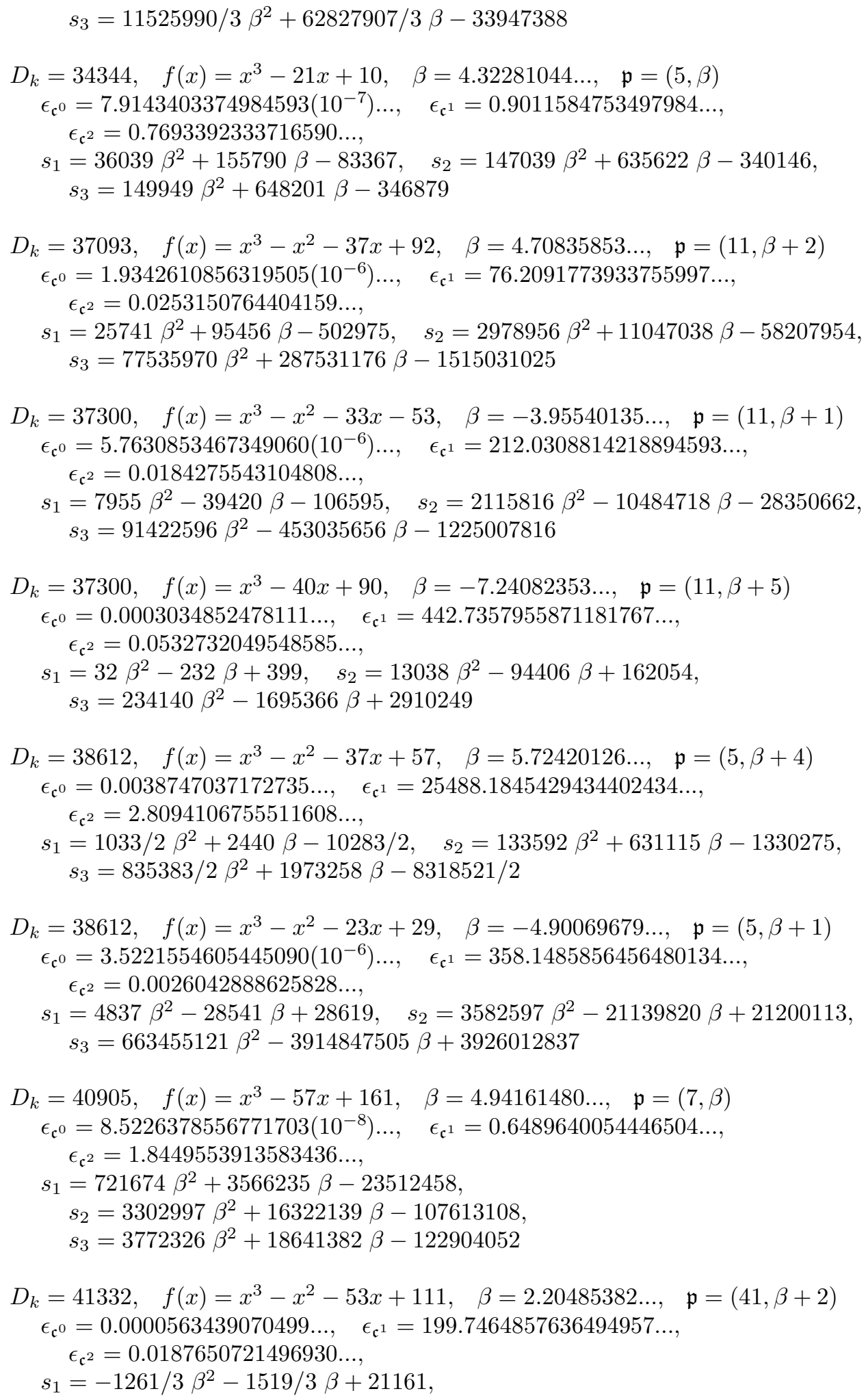




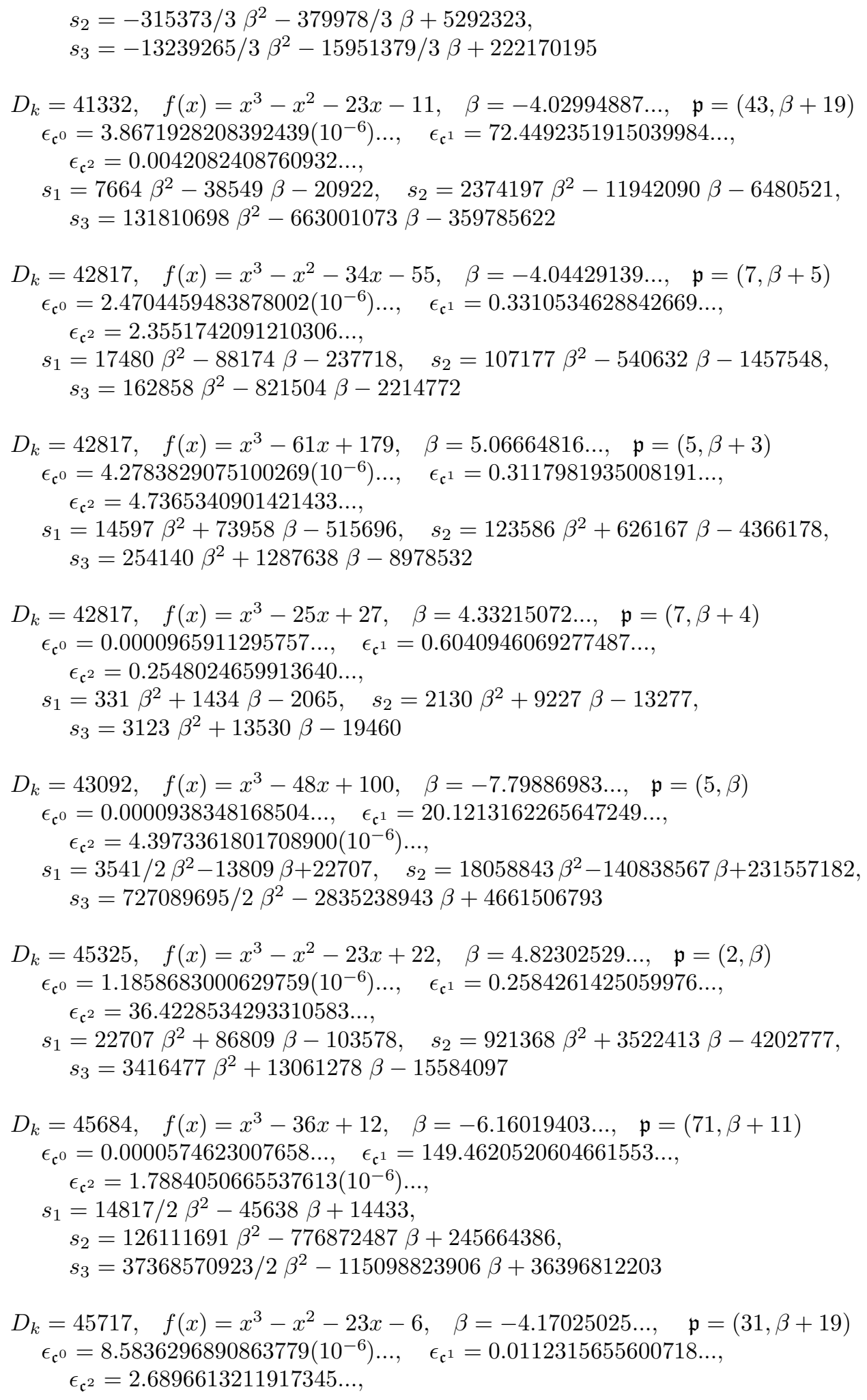


1264 DAVID S. DUMMIT, JONATHAN W. SANDS, AND BRETT A. TANGEDAL

$$
\begin{gathered}
s_{1}=3108 \beta^{2}-16069 \beta-4470, \quad s_{2}=286054 \beta^{2}-1478970 \beta-411567, \\
s_{3}=846612 \beta^{2}-4377195 \beta-1218079 \\
D_{k}=46548, \quad f(x)=x^{3}-60 x+174, \quad \beta=5.06271682 \ldots, \quad \mathfrak{p}=(11, \beta+2) \\
\epsilon_{\mathfrak{c}^{0}}=2.6671937837242204\left(10^{-10}\right) \ldots, \quad \epsilon_{\mathfrak{c}^{1}}=27.3003706065528768 \ldots, \\
\epsilon_{\mathfrak{c}^{2}}=0.0080919034750920 \ldots, \\
s_{1}=221937571 \beta^{2}+1123607074 \beta-7627749828, \\
s_{2}=33496017624 \beta^{2}+169580851862 \beta-1151221226200, \\
s_{3}=749824123748 \beta^{2}+3796147204096 \beta-25770629119676 \\
D_{k}=46548, \quad f(x)=x^{3}-36 x+4, \quad \beta=-6.05480244 \ldots, \quad \mathfrak{p}=(5, \beta+3) \\
\epsilon_{\mathfrak{c}^{0}}=4.0234729424478067\left(10^{-10}\right) \ldots, \quad \epsilon_{\mathfrak{c}^{1}}=48.5936439176364186 \ldots, \\
\epsilon_{\mathfrak{c}^{2}}=0.2505274973657315 \ldots, \\
s_{1}=33594908 \beta^{2}-203410531 \beta+22193892, \\
s_{2}=1775703443 \beta^{2}-10751533544 \beta+1173087616, \\
s_{3}=6928165268 \beta^{2}-41948671988 \beta+4576971972
\end{gathered}
$$

$D_{k}=46644, \quad f(x)=x^{3}-x^{2}-69 x-183, \quad \beta=-5.49617539 \ldots, \quad \mathfrak{p}=(3, \beta)$

$\epsilon_{\mathfrak{c}^{0}}=0.0007480433912605 \ldots, \quad \epsilon_{\mathfrak{c}^{1}}=233814.3715515913653399 \ldots$,

$\epsilon_{\mathfrak{c}^{2}}=0.1200570746203687 \ldots$

$s_{1}=7210 \beta^{2}-46837 \beta-240064, \quad s_{2}=9644139 \beta^{2}-62650018 \beta-321110103$, $s_{3}=80972747 \beta^{2}-526013167 \beta-2696058917$
$D_{k}=46813, \quad f(x)=x^{3}-x^{2}-43 x+116, \quad \beta=-7.20403114 \ldots, \quad \mathfrak{p}=(17, \beta+15)$
$\epsilon_{\mathfrak{c}^{0}}=7.6001787555541085\left(10^{-7}\right) \ldots, \quad \epsilon_{\mathfrak{c}^{1}}=0.0110341869090405 \ldots$, $\epsilon_{\mathfrak{c}^{2}}=3.1596420064362269 \ldots$
$s_{1}=10353 \beta^{2}-84933 \beta+166692, \quad s_{2}=974274 \beta^{2}-7992972 \beta+15687845$, $s_{3}=3261609 \beta^{2}-26758344 \beta+52518751$
$D_{k}=47860, \quad f(x)=x^{3}-x^{2}-61 x+185, \quad \beta=6.02818494 \ldots, \quad \mathfrak{p}=(5, \beta+2)$
$\epsilon_{\mathfrak{c}^{0}}=0.0004587112066891 \ldots, \quad \epsilon_{\mathfrak{c}^{1}}=385948.0096909855103361 \ldots$, $\epsilon_{\mathfrak{c}^{2}}=0.0617103648674198 \ldots$ $s_{1}=21587 / 2 \beta^{2}+54272 \beta-662485 / 2$, $s_{2}=23572651 \beta^{2}+118527649 \beta-723425124$, $s_{3}=761174735 / 2 \beta^{2}+1913663670 \beta-23359821793 / 2$


It remains to compute explicitly the Frobenius element $\sigma_{\mathfrak{c}}$. This can be done with the data provided above, as described in Section 5, but is rather more intensive, unlike the simple computations in (a)-(c) above. As a result we include below the explicit Frobenius automorphisms only for the 5 fields with discriminant less than 10000 and for the field of discriminant 28212 described in Section 6. The data for the remaining examples as well as electronic versions of the data in these tables can be obtained by email from the authors.

$$
\begin{aligned}
& D_{k}=2597 \\
& \sigma_{\mathfrak{c}}(\epsilon)=[ \\
&\left(25328-1356 \beta-3364 \beta^{2}\right) \epsilon^{5}+ \\
&\left(-137616+28858 \beta+25252 \beta^{2}\right) \epsilon^{4}+ \\
&\left(318696-98190 \beta-69273 \beta^{2}\right) \epsilon^{3}+ \\
&\left(-358073+112785 \beta+79331 \beta^{2}\right) \epsilon^{2}+ \\
&\left.\left(199168-39527 \beta-36246 \beta^{2}\right) \epsilon+\left(-51496+1845 \beta+6724 \beta^{2}\right)\right] / 3997
\end{aligned}
$$

$D_{k}=4212$

$$
\begin{aligned}
\sigma_{\mathfrak{c}}(\epsilon)=[ & \left(-340775+464198 \beta+320869 \beta^{2}\right) \epsilon^{5}+ \\
& \left(40526716-34401038 \beta-11816512 \beta^{2}\right) \epsilon^{4}+ \\
& \left(-235405572+202535726 b \beta+69063980 \beta^{2}\right) \epsilon^{3}+ \\
& \left(411620440-353633098 \beta-120602498 \beta^{2}\right) \epsilon^{2}+ \\
& \left(-128401572+110581112 \beta+37797938 \beta^{2}\right) \epsilon+ \\
& \left.\left(3039548-2230368 \beta-923186 \beta^{2}\right)\right] / 610109
\end{aligned}
$$

$$
\begin{aligned}
& D_{k}=6885 \\
& \sigma_{\mathfrak{c}}(\epsilon)=\left[\left(-154+747 \beta+225 \beta^{2}\right) \epsilon^{5}+\left(50+2991 \beta+905 \beta^{2}\right) \epsilon^{4}+\right. \\
& \left(-969+5746 \beta+1547 \beta^{2}\right) \epsilon^{3}+\left(85+4743 \beta+1513 \beta^{2}\right) \epsilon^{2}+ \\
& \left.\left(-509+2517 \beta+710 \beta^{2}\right) \epsilon+\left(69+477 \beta+132 \beta^{2}\right)\right] / 17
\end{aligned}
$$

$D_{k}=9653$

$$
\begin{aligned}
\sigma_{\mathfrak{c}}(\epsilon)=[ & \left(136280-62660 \beta-28884 \beta^{2}\right) \epsilon^{5}+ \\
& \left(823150-1571890 \beta+310096 \beta^{2}\right) \epsilon^{4}+ \\
& \left(-1646711+3813888 \beta-1079473 \beta^{2}\right) \epsilon^{3}+ \\
& \left(1666493-3533513 \beta+788409 \beta^{2}\right) \epsilon^{2}+ \\
& \left(-437163+794029 \beta-263140 \beta^{2}\right) \epsilon+ \\
& \left.\left(130581-93101 \beta-8590 \beta^{2}\right)\right] / 191681
\end{aligned}
$$

$$
\begin{aligned}
D_{k}=9800 & \\
\sigma_{\mathfrak{c}}(\epsilon)=[ & \left(22571911+36645874 \beta-1370309 \beta^{2}\right) \epsilon^{5}+ \\
& \left(-63256308-33101528 \beta+6061580 \beta^{2}\right) \epsilon^{4}+ \\
& \left(-219770863-5441722 \beta+16330639 \beta^{2}\right) \epsilon^{3}+ \\
& \left(2852220080+204247608 \beta-133579400 \beta^{2}\right) \epsilon^{2}+ \\
& \left(-2000686116-103046736 \beta+89250564 \beta^{2}\right) \epsilon+ \\
& \left.\left(280105064-14215168 \beta-10318120 \beta^{2}\right)\right] / 47722922
\end{aligned}
$$




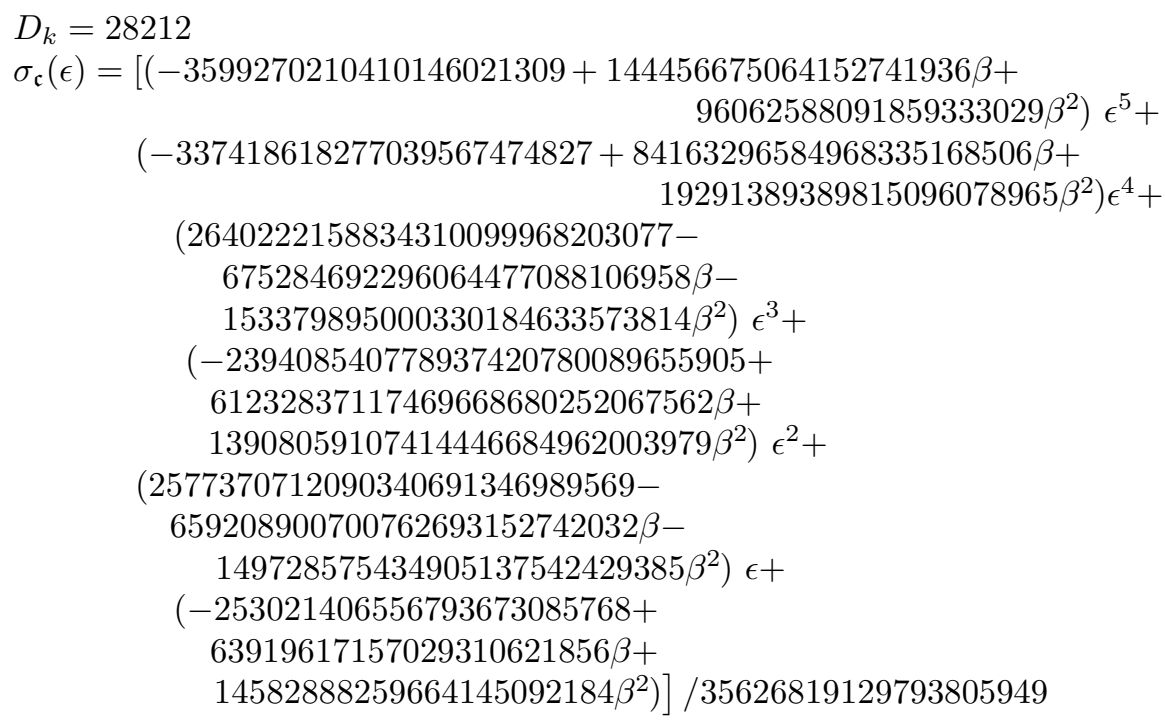

\section{REFERENCES}

1. T. Arakawa, Generalized eta-functions and certain ray class invariants of real quadratic fields, Math. Ann. 260 (1982), 475-494. MR 84b:12016

2. B. L. J. Braaksma, Asymptotic expansions and analytic continuations for a class of Barnesintegrals, Comp. Math. 15 (1963), 239-341. MR 29:4923

3. D. S. Dummit and D. Hayes, Checking the refined $\mathfrak{p}$-adic Stark Conjecture when $\mathfrak{p}$ is Archimedean, Algorithmic Number Theory, Proceedings ANTS 2, Talence, France, Lecture Notes in Computer Science 1122 (Henri Cohen, ed.), Springer-Verlag, Berlin-Heidelberg-New York, 1996, pp. 91-97.

4. V. Ennola and R. Turunen, On totally real cubic fields, Math. Comp 44 (170) (1985), 495-518. MR 86e: 11100

5. C. Fogel, personal communication.

6. E. Friedman, Hecke's Integral Formula, Séminaire de Théorie des Nombres de Bordeaux No. 5 (1987-88) (1989). MR 90i:11136

7. F. Hajir, Elliptic units of cyclic unramified extensions of complex quadratic fields, Acta Arithmetica 64 (1993), 69-85. MR 94h:11102

8. F. Hajir, Unramified Elliptic Units, M.I.T. Thesis, 1993.

9. F. Hajir (with F. Rodriguez-Villegas), Explicit Elliptic Units I, Duke Math. J. (to appear).

10. D.R. Hayes, Brumer elements over a real quadratic base field, Expositiones Mathematicae 8 (1990), 137-184. MR 92a:11142

11. D.R. Hayes, The partial zeta functions of a real quadratic field evaluated at $s=0$, Number Theory (Richard A. Mollin, ed.), Walter de Gruyter, Berlin, 1990, pp. 207-226. MR 92j:11134

12. D.R. Hayes, Base Change for the Brumer-Stark Conjecture, preprint.

13. J. Nakagawa, On the Stark-Shintani conjecture and cyclotomic $\mathbb{Z}_{p}$-extensions of class fields over real quadratic fields, J. Math. Soc. Japan 36 (4) (1984), 577-588. MR 87a:11108a

14. K. Rubin, A Stark Conjecture "over $\mathbb{Z}$ " for abelian L-functions with multiple zeros, Ann. Inst. Fourier, Grenoble 46 (1996), 33-62. CMP 96:11

15. J. W. Sands, Abelian fields and the Brumer-Stark conjecture, Comp. Math. 53 (1984), 337346. MR 86c: 11102

16. J. W. Sands, Galois groups of exponent two and the Brumer-Stark conjecture, J. Reine Angew. Math. 349 (1984), 129-135. MR 85i:11098

17. J. W. Sands, Two cases of Stark's conjecture, Math. Ann. 272 (1985), 349-359. MR 87a: 11117

18. T. Shintani, On a Kronecker limit formula for real quadratic fields, J. Fac. Sci. Univ. Tokyo 24 (1977), 167-199. MR 57:277 
19. T. Shintani, On certain ray class invariants of real quadratic fields, J. Math. Soc. Japan 30 (1978), 139-167. MR 58:16599

20. H. M. Stark, Class fields for real quadratic fields and L-series at 1, Algebraic Number Fields (A. Fröhlich, ed.), Academic Press, London, 1977, pp. 355-375. MR 56:11963

21. H. M. Stark, Hilbert's twelfth problem and L-series, Bull. A.M.S. 83 (5) (1977), 1072-1074. MR 56:314

22. H. M. Stark, Values of L-functions at $s=1$. I. L-functions for quadratic forms, Advances in Math. 7 (1971), 301-343. MR 44:6620

23. H. M. Stark, Values of L-functions at $s=1$. II. Artin L-functions with rational characters, Advances in Math. 17 (1975), 60-92. MR 52:3082

24. H. M. Stark, L-functions at $s=1$. III. Totally real fields and Hilbert's twelfth problem, Advances in Math. 22 (1976), 64-84. MR 55:10427

25. H. M. Stark, L-functions at $s=1 \mathrm{IV}$. First derivatives at $s=0$, Advances in Math. 35 (1980), 197-235. MR 81f: 10054

26. J. T. Tate, Les conjectures de Stark sur les fonctions $L$ d'Artin en $s=0$, Birkhäuser, Boston, 1984. MR 86e: 11112

27. F. Y. Wang, Conductors of fields arising from Stark's conjecture, Ph.D. Thesis, MIT, 1991.

28. A. Wiles, On a conjecture of Brumer, Annals of Math. 131 (1990), 555-565. MR 91i:11164

Department of Mathematics and Statistics, University of Vermont, Burlington, VerMONT 05405

E-mail address: dummit@math.uvm.edu 